No. 21-17

\title{
Did the Affordable Care Act Affect Access to Medications for Opioid Use Disorder among the Already Insured? Evidence from the Rhode Island All-payer Claims Database
}

\author{
Mary A. Burke, Katherine Carman, Riley Sullivan, Hefei Wen, J. Frank Wharam, and Hao Yu
}

\begin{abstract}
:
Previous research suggests that state Medicaid expansions implemented under the Patient Protection and Affordable Care Act (ACA) helped large numbers of patients suffering from opioid use disorder (OUD) gain access to life-saving medications, including buprenorphine. However, Medicaid expansions could have impeded access to care among individuals already enrolled in Medicaid, as new enrollees would have placed added demands on a limited supply of buprenorphine providers. Using a panel data set of medical claims from Rhode Island, we estimate the causal effects of the state's January 2014 ACA implementation on buprenorphine receipt among incumbent (pre-ACA) Medicaid enrollees by leveraging geographic variation within Rhode Island in the intensity of treatment under the ACA. Using a difference-in-differences identification strategy, multivariate regression analysis yields no evidence that incumbent Medicaid enrollees experienced added difficulties in accessing buprenorphine as a result of the ACA, despite the fact that both Medicaid and nonMedicaid enrollment increased substantially under the policy. Supply-side factors may have helped to blunt any negative fallout, as we find that the number of buprenorphine prescribers in the state increased fairly steadily during the two years leading up to January 2014 and for at least 15 months after that date. Also, the average number of buprenorphine recipients per prescriber increased rapidly after January 2014, suggesting that providers had unused treatment capacity before the policy went into effect.
\end{abstract}

JEL Classifications: I18, I13, I14, I12

Keywords: Medicaid expansion, opioid use disorder (OUD), methadone, buprenorphine, Rhode Island, all-payer claims database

Mary A. Burke (Mary.Burke@bos.frb.org) is a senior economist and policy advisor with the New England Public Policy Center (NEPPC) in the Federal Reserve Bank of Boston Research Department. Katherine Carman (Katherine_Carman@rand.org) is a senior economist at the RAND Corporation. Riley Sullivan (Riley.Sullivan@bos.frb.org) is a senior policy analyst with the NEPPC. Hefei Wen (hefei_wen@hphci.harvard.edu) is an assistant professor in the Department of Population Medicine (DPM) at the Harvard Pilgrim Health Care Institute and Harvard Medical School (HMS). J. Frank Wharam (jwharam@post.harvard.edu) and Hao Yu (hao_yu@hphci.harvard.edu) are associate professors in the DPM and HMS.

The authors thank Samuel Makikalli, Morgan Klaeser, David Schramm, and Melissa Gentry for excellent research assistance and data analysis. They are grateful to Brian Boates, Jeffrey Thompson, Darcy Saas, Delia Sawhney, Jones George, and Bret Fontecchio for their help in acquiring and securing the data. The authors thank Catherine Maclean and other participants at the 10th annual conference of the American Society of Health Economists for many helpful suggestions. They also thank Jamieson Goulet of the Rhode Island Department of Behavioral Health, Developmental Disabilities, and Hospitals for answering policy-related questions.

Data for this analysis were obtained through an approved request to HealthFacts RI, Rhode Island's all-payer claims database, as administered by the Rhode Island Department of Health (RIDOH). Data were obtained for the period April 2011 through May 2019. $\mathrm{RIDOH}$ is not responsible for the authors' analysis, opinions, and conclusions contained in this document.

The views expressed herein are those of the authors and do not indicate concurrence by the Federal Reserve Bank of Boston, the principals of the Board of Governors, or the Federal Reserve System. This paper, which may be revised, is available on the website of the Federal Reserve Bank of Boston at https://www.bostonfed.org/publications/research-department-working-paper.aspx. 
The high prevalence of opioid use disorder (OUD) in the United States remains an urgent public health concern. ${ }^{1}$ Opioid-related mortality in the United States experienced a sharp resurgence in 2020 amid the COVID-19 pandemic. The 2020 surge followed a three-year period (2017 through 2019) yielding flat or declining opioid death rates that were nonetheless substantially elevated in historical terms. ${ }^{2}$ OUD not only contributes to premature mortality but also impairs social functioning and carries external costs such as increased crime, increased disease transmission, and reduced productivity (Wall et al. 2000; Florence, Luo, and Rice 2021). Effective medications for OUD are available, and yet many or even most OUD patients do not receive them (Saloner and Karthikeyan 2015). These evidence-based medications - referred to collectively as medications for addiction treatment (MAT) or medications for opioid use disorder (MOUD) consist of administering any of the U.S. Food and Drug Administration (FDA)-approved medications methadone, buprenorphine, or naltrexone, typically in conjunction with behavioral therapy. ${ }^{3}$ Treatment proceeds with an initial detoxification phase that is followed by a maintenance phase involving the use of one of these medications for an extended time period (Schuckit 2016).

The barriers to utilization of MOUD are numerous and complex. They include, but are not limited to, regulations that restrict the supply of these medications, gaps in health insurance coverage, and social stigmas against using opioid agonist medications (a class that includes both methadone and buprenorphine) to treat OUD. Regarding health insurance, Medicaid has been cited as an important source of access to MOUD, as the populations targeted by Medicaid tend to

\footnotetext{
${ }^{1}$ The DSM-5 (Diagnostic and Statistical Manual of Mental Disorders, Fifth Edition) defines an OUD as "a problematic pattern of opioid use that leads to serious impairment or distress." (American Psychiatric Association 2013). The condition is also characterized as a chronic brain disease rooted in neurobiology (Volkow et al. 2014). ${ }^{2}$ More than 40 states reported increases in opioid-related fatalities compared with the same period in 2019. See American Medical Association, "Reports of Increases in Opioid- and Other Drug-related Overdose and Other Concerns during COVID Pandemic," December 9, 2020. https://www.ama-assn.org/system/files/2020-12/issuebrief-increases-in-opioid-related-overdose.pdf

${ }^{3}$ MAT is strongly endorsed by the World Health Organization and the United States Department of Health and Human Services. Through decades of clinical research, MAT has been proven effective in reducing abuse of opioids, risk of fatal overdose, and all-cause mortality (see, for example, Gibson et al. 2008 and Connery 2015). In addition to its benefits for individual OUD patients, MAT has been found to offer broader benefits to public health, such as reductions in HIV and hepatitis C risk behaviors as well as reductions in criminal behavior (Evans et al. 2019). Observational studies from two New England states, Massachusetts (Larochelle et al. 2018) and Vermont (Mohlman et al. 2016), find, respectively, that MAT is associated with lower risk of fatal opioid overdose and lower health-care expenditures.
} 
show a high prevalence of severe OUD (Donohue et al. 2019) and many state Medicaid plans offer generous coverage for medications for OUD, including either buprenorphine or methadone or both drugs. Indeed, previous research suggests that state Medicaid expansions implemented starting in 2014 under the Patient Protection and Affordable Care Act (ACA) helped large numbers of OUD patients gain access to MOUD that was previously lacking, potentially saving and improving lives as a result (Wen et al. 2017; Clemans-Cope, Epstein, and Kenney 2017; Meinhofer and Witman 2018). However, Maclean and Saloner (2019) find that admissions to specialty treatment facilities for all types of substance use disorders (not just OUD) did not increase significantly in the aggregate owing to the Medicaid expansion, but instead payments for such admissions shifted to Medicaid from state and local funds targeting the uninsured.

One potential drawback of state Medicaid expansion policies concerns the impact on individuals who were already enrolled in Medicaid prior to the implementation of these health insurance expansions. According to the Kansas Policy Institute, pre-expansion Medicaid enrollees (such as pregnant women, disabled persons, children, and very low-income families) "are at risk of being pushed to the back of the line" in order to accommodate expansion enrollees - the latter being poor but childless adults. ${ }^{4}$ At least three studies raise the concern that Medicaid expansions would have placed added demands on a limited supply of providers of either buprenorphine (Wen et al. 2017) or specialty substance abuse treatment (Grooms and Ortega 2019; Meinhofer and Witman 2018), potentially creating bottlenecks in access to care. However, Grooms and Ortega (2019) find that Medicaid expansions were not associated with any increase in wait times to enter specialty treatment facilities for substance use disorders. Related to that outcome, Meinhofer and Witman (2018) find that the ACA (and especially Medicaid expansions) induced increases in the supply of substance use treatment facilities accepting Medicaid, a response that may have precluded congestion effects. Further emphasizing the importance of the supply side, Wen et al. (2017) find that a state's buprenorphine provider count was positively associated with its increase in Medicaid-paid buprenorphine prescriptions following Medicaid expansion.

However, none of the previous studies follows outcomes among Medicaid incumbents specifically, and so their findings offer no direct evidence concerning incumbent enrollees'

\footnotetext{
${ }^{4}$ The Kansas Policy Institute is a conservative-leaning think tank based in Wichita. URL: https://kansaspolicy.org/medicaid-expansion-hurts-truly-needy/, site accessed October 3, 2021.
} 
access to MOUD following Medicaid expansions. Furthermore, as those studies all employ a similar research design contrasting changes in statewide outcomes in Medicaid expansion states against those in non-expansion states, their results are likely driven primarily by the experience of people who first entered Medicaid under their states' expansion policies (as explained, for example, in Courtemanche et al. 2017). Considering health-care services in general, not specifically treatment for OUD, research regarding the impact of Medicaid expansions on appointment wait times and other access-to-care indicators is relatively scarce, and so far, only a single study observes a significant increase in wait times (Miller and Wherry 2017). A review article (Mazurenko et al. 2018) concludes that there is a need for more research that addresses the question of whether "an increase in health care use among new Medicaid enrollees has a net overall beneficial effect for people and the system at large."

Using the all-payer claims database (APCD) for Rhode Island (or HealthFactsRI) spanning the period January 2012 through December 2015, we test directly for whether incumbent (pre-2014) Medicaid enrollees in Rhode Island experienced any systematic changes in their use of buprenorphine after January 2014, the month in which the state implemented the Affordable Care Act and its associated Medicaid expansion. The interruption or delay of buprenorphine treatment among those in need of MOUD could carry serious health consequences, as previous research (Burke et al. 2020) finds that recent adherence to buprenorphine treatment reduced the chances of nonfatal opioid overdose relative to having ever received the drug but not taking it recently. We also examine changes in the number of buprenorphine prescribers in Rhode Island and in their patient loads before and after January 2014 to gain additional insights into the ACA's potential implications for incumbent patients. This study is among the first to directly address either the incumbent enrollee experience with buprenorphine utilization under the ACA or changes in buprenorphine prescribing capacity under the ACA. It also adds to a relatively small group of studies that consider post-ACA outcomes among incumbents along any dimension of care. We can't perfectly separate the effects of the Medicaid expansion from the larger ACA, as the policies were implemented simultaneously, but we do compare the effects of increased Medicaid enrollment after 2014 separately from the effects of increased enrollment in non-Medicaid plans after 2014. 
In an attempt to identify causal effects on buprenorphine receipt among Medicaid incumbents, we leverage geographic variation within Rhode Island in the intensity of treatment under the ACA, using three different measures of treatment intensity: increased Medicaid enrollment in 2014 and 2015 by county subdivision, increased non-Medicaid enrollment in 2014 and 2015 by county subdivision, and uninsurance rates by county subdivision for the pre-treatment (20092013 ) period. The uninsurance rates are employed as exogenous proxies for the realized, postACA enrollment growth rates by county subdivision, as higher pre-ACA uninsurance rates are found to predict greater enrollment growth in the post-ACA period. The methods used are adapted from those of Miller (2012), who exploits within-state variation in Massachusetts in the intensity of treatment under that state's 2006 health insurance reform to identify the effects of that reform on emergency room visits. Similarly, Courtemanche et al. (2017) exploit both crossstate and within-state variation in the intensity of policy treatment to study the response of health insurance enrollment to ACA implementation and Medicaid expansion, and Finkelstein (2007) uses a similar identification strategy to measure the effects of Medicare introduction on healthcare spending.

We find that the rate of buprenorphine receipt within a stable group of Medicaid incumbents generally increased from January 2012 to December 2015, although the pace of increase was slower in the post-ACA period (January 2014 through December 2015) compared with the earlier period. The slower growth pace after January 2014 suggests that implementation of the ACA - under which a large number of individuals gained access to health insurance coverage for substance abuse treatment including (in many cases) buprenorphine for OUD — might have made it harder for incumbent Medicaid enrollees to fill buprenorphine prescriptions. To consider just one potential mechanism, patients taking buprenorphine for OUD are typically required to visit their provider at least once per month, or even on a daily or weekly basis in the initial phase of treatment, in order to secure additional doses and to undergo monitoring and/or counselling (Langabeer et al. 2020; Yarbrough, Abraham, and Adams et al. 2020). Any delays in securing an appointment could result in an interruption of treatment. Practices that took on new enrollees after the ACA might have had fewer remaining appointments for incumbents, making it harder to secure a convenient slot. 
If the aggregate pattern was in fact caused by the ACA, then any headwinds against buprenorphine utilization arguably should have been felt most acutely in the places in Rhode Island that experienced the greatest influxes of new enrollees under the ACA, all else being equal. However, multivariate regression analysis yields no evidence of such a pattern. Instead, we find that Medicaid incumbents in county subdivisions (which correspond to cities or towns) that were subject to larger (relative) influxes of new (Medicaid or non-Medicaid) enrollees in 2014 and 2015 experienced roughly equivalent or possibly larger increases following the ACA in their chances of receiving buprenorphine. These results control for numerous other factors that predict buprenorphine receipt, such as county subdivision fixed effects, aggregate time trends, and individual health status indicators. Results are qualitatively robust to using pre-2014 uninsurance rates by county subdivision as the measure of treatment intensity in order to address the endogeneity of the actual enrollment increases, and to adopting a conditional logit (individual fixed effects) model. Sensitivity analysis reveals further that any positive associations between the intensity of treatment under the ACA and subsequent gains in buprenorphine use are probably not causal but instead reflect differences in pre-ACA trends across different county subdivisions. Nonetheless, the sensitivity analysis still offers no evidence of negative effects of the ACA on buprenorphine use among incumbents.

Regarding the supply-side response, the number of buprenorphine providers treating Rhode Island patients in our sample - both overall and the number treating Medicaid patients specifically — increased fairly steadily over the 2012-2014 period, but then stabilized in 2015. The net increase in providers from 2012-2013 to 2014-2015 was modest in comparison with the overall surge in health insurance enrollees (of any insurance type) over the same period. Accordingly, we observe that buprenorphine providers on average assumed higher patient loads starting in 2014, and in particular, the number of Medicaid patients per provider increased markedly (not restricting to incumbent patients). To reiterate, our analysis suggests that any negative impact on incumbents of an increase in patient loads per provider shows up (if at all) only in the aggregate and does not align with our measures of treatment intensity. 
One explanation for a (presumed) lack of negative effects would be that, whether before or after the ACA, the average provider in Rhode Island appeared to operate with excess capacity. ${ }^{5}$ Prior to 2014 , the average provider served roughly 12 or 13 patients in a given month (considering all insurance plans) and fewer than seven Medicaid patients, whereas the maximum patient limit per provider (as of 2014) was 30 patients per provider in most cases and as many as 100 patients for some providers. Even after 2014, average patient loads per provider per month stayed well below these legal limits, rising to at most 18 total patients and 12 Medicaid patients. On the other hand, after 2014 some providers may have edged closer to their effective capacity constraints, which may have fallen short of the legal limit but which can't be observed. It is therefore possible that patients faced longer wait times for appointments or found it harder to match with a provider starting in early 2014, and such prescribing constraints may have contributed to the aggregate slowdown in the growth rate of buprenorphine utilization among Medicaid incumbents after ACA implementation.

The remainder of the paper is organized as follows. Section 2 provides background information on Rhode Island's Medicaid program, the progression of the state's opioid crisis, and its policy responses. Section 3 discusses the data and describes aggregate outcomes. Section 4 describes the empirical model and identification strategy. Section 5 presents the regression results. Section 6 describes changes in provider supply. Section 7 interprets the results and discusses the potential policy implications.

\section{Background: Rhode Island's Opioid Crisis, Medicaid Program, and ACA Implementation}

Rhode Island represents an important object of study for understanding the opioid crisis and potential policy solutions. Since the early 2000s, the state has experienced above-average rates of opioid-associated mortality, and in 2018 its opioid-related overdose death rate was 10th highest in the United States. ${ }^{6}$ Like the country as a whole, the state experienced a resurgence in opioid deaths in 2020. In that year, Rhode Island's opioid-related mortality rate per 100,000 persons hit a record high of 30.6, despite having fallen to 24.2 in 2019 from its previous record of 27.4

\footnotetext{
${ }^{5}$ Evidence on buprenorphine prescribing from several other states yields similar findings (see, for example, Stein et al. 2016).

${ }^{6}$ This ranking was calculated by the Centers for Disease Control and Prevention using data from 38 states. Three other New England states - New Hampshire, Massachusetts, and Connecticut—had even higher opioid-related death rates per capita than Rhode Island in 2018 (Kaiser Family Foundation 2020).
} 
(reached in 2016). The 2020 figure is roughly triple the state's 2010 opioid death rate of $10.2 .^{7}$ Since at least 2015, when then-Governor Gina Raimondo established the state's Overdose Prevention and Intervention Task Force, Rhode Island has been a national leader in advancing policies to promote the use of medications for opioid use disorder (MOUD). ${ }^{8}$ Previous research suggests that the state has achieved an above-average uptake of MOUD compared with other US states (Burke 2019), and that Rhode Island health insurance enrollees taking either buprenorphine or methadone faced lower risks of having a (nonfatal) opioid overdose compared with otherwise similar but untreated patients (Burke et al. 2021).

In an earlier policy move affecting access to MOUD, Rhode Island expanded its Medicaid program as part of its implementation of the federal Patient Protection and Affordable Care Act (ACA), all of which went into effect in the state in January 2014. Under the state's expansion, eligibility for Medicaid was extended to all non-disabled adults aged 18 to 64 (with or without children) with incomes at or below 138 percent of the federal poverty level. Previously, aside from low-income disabled individuals and low-income elderly individuals, only parents with family income up to 175 percent of the poverty level and pregnant women and children in families with income up to 250 percent of the poverty level were eligible for Medicaid. ${ }^{9}$ Note that parents with family incomes ranging from 138 percent to 175 percent of the poverty level lost eligibility for Medicaid in Rhode Island in 2014. This loss was compensated by the availability of subsidized insurance under the state's health insurance exchange, which was also launched in 2014 as part of ACA implementation. Including adults and children, Medicaid enrollment in Rhode Island swelled nearly 20 percent from fiscal year 2014 to fiscal $2015 .{ }^{10}$ As of 2019, 24 percent of Rhode Island's population (including children) was covered by Medicaid. ${ }^{11}$

\footnotetext{
${ }^{7}$ Source: Prevent Overdose RI, "Overdose Death Data," www.preventoverdoseri.org/overdose-deaths.

${ }^{8}$ In 2016, Rhode Island became the first state to offer MAT to prison inmates and subsequently saw a steep decline in the number of overdose deaths among former inmates (Green et al. 2018). In 2017, the state formed a partnership with Brown University's Warren Alpert Medical School to incorporate training in addiction treatment into the curriculum, providing a model for a federal program enacted in 2018.

${ }^{9}$ For an extended discussion of Rhode Island's actions in advance of the ACA and Medicaid expansion, see Holahan et al. (2012).

${ }^{10}$ See BallotPedia, "Effect of the Affordable Care Act in Rhode Island (2009-2017)," https://ballotpedia.org/Effect of the Affordable Care Act in Rhode Island, accessed June 2, 2021.

${ }^{11}$ See Kaiser Family Foundation, "Medicaid in Rhode Island" fact sheet, https://files.kff.org/attachment/fact-sheetmedicaid-state-RI, accessed June 2, 2021.
} 
Most disabled and elderly Medicaid enrollees in Rhode Island participate in the state-run fee-forservice plan, whereas most non-disabled, non-elderly enrollees are assigned to the RIte Care program, which offers a choice of managed care from one of three private insurance companies - as of 2014 the state had only two managed care plans; the third entered the state in 2018. ${ }^{12}$ As of 2012, Medicaid plans in Rhode Island, whether fee-for-service or managed care, covered medications for opioid use disorder, including both methadone, as dispensed at an opioid treatment programs (OTP), and buprenorphine, as prescribed from a qualified officebased practitioner. This coverage entailed no out-of-pocket payments for either medication, and buprenorphine prescriptions were not subject to prior authorization requirements. ${ }^{13}$ Given these facts, the ACA's revised mental health parity and essential health benefits requirements - which went into effect in January 2014 - would not have imposed any binding changes in the coverage of OUD treatment, and buprenorphine utilization in particular, on the part of the state's Medicaid managed care plans (and did not apply to the state's fee-for-service plan).

Rhode Island also adopted the ACA's optional Medicaid health home model for individuals with opioid use disorder, which went into effect in July 2013. The health home model emphasizes a coordinated care approach that integrates the use of medications for OUD with behavioral health care and other services. ${ }^{14}$ All specialized OTPs in Rhode Island - which overwhelmingly administer methadone for OUD rather than buprenorphine - were required to participate, while office-based prescribers of buprenorphine were not part of the state's health home model (Clemans-Cope, Epstein, and Kenney 2017). This additional policy treatment, which went into effect shortly before ACA implementation, presents difficulties in assessing the effects of the subsequent ACA on methadone use. For this and other data-related reasons, the analysis in this paper focuses on buprenorphine utilization rather than on methadone use or simply any MOUD use. The limitations of this approach are discussed in Section 7 below.

\section{Data and Methods}

\footnotetext{
${ }^{12}$ See Schneider et al. (2002) and https://eohhs.ri.gov/consumer/families-children/healthcare-programs.

${ }^{13}$ Despite the lack of prior authorization requirement, the buprenorphine formulations used to treat OUD did not appear on the preferred drug list of Medicaid fee-for-service in Rhode Island as of 2012. See Burns et al. (2016) and https://eohhs.ri.gov/sites/g/files/xkgbur226/files/Portals/0/Uploads/Documents/Pharmacy/pdl_list.pdf .

${ }^{14}$ For more information about Rhode Island's OTP Health Home model, see https://www.medicaid.gov/stateresource-center/innovation-accelerator-program/iap-downloads/reducing-substance-use-disorders/mat-key-elementsri.pdf.
} 


\section{3a. Data Overview}

HealthFacts RI is the official name of the all-payer claims database (APCD) for the state of Rhode Island. ${ }^{15}$ The Federal Reserve Bank of Boston entered into a data-use agreement with the Rhode Island Department of Health, and the research received institutional review board approval and was deemed exempt from human subjects research protocols. The database in our possession is organized at the monthly frequency and runs from April 2011 through May 2019, although we use a narrower range of dates in the analysis sample for reasons discussed below. For each health insurance enrollee observed in a given month, the database provides age, gender, and residential Zip code; information about their medical and/or pharmacy insurance plans; and extensive information from insurance claims pertaining to the health-care services and prescription medications the enrollee received in that month. ${ }^{16}$ The data have been de-identified to protect confidentiality, but individual enrollees and health-care providers can be followed over time using numerical identifiers. Data on race and ethnicity are not available.

Plans that insure fewer than 3,000 Rhode Island residents or employer-based plans that insure fewer than 3,000 individuals who work in Rhode Island (regardless of where they live) are not required to report to the APCD. Starting in March of 2016, self-insured health plans covered by the ERISA (Employee Retirement Income Security Act) law were no longer required to report to the APCD, and this results in reduced coverage of commercial enrollees in our sample at around that time. ${ }^{17}$ From mid-2011 through mid-2018, the data capture from 76 percent to 88 percent of Rhode Island residents with health insurance; that number peaked between mid-2014 and mid-

\footnotetext{
${ }^{15}$ Currently, 18 states have full APCDs, and 12 more either have voluntary APCDs not mandated by legislation or they are still in the process of establishing the APCDs. See the Agency for Healthcare Quality and Research for more information, https://www.ahrq.gov/data/apcd/index.html.

${ }^{16}$ To cite just a few data items, we observe detailed codes and descriptions of all procedures, treatments, and diagnoses; standardized drug codes and the quantity supplied for each prescription; payment information such as the enrollee's copay and the full amount charged for each service or medication; and information about the provider who rendered the services.

${ }^{17}$ In March 2016, the Supreme Court ruled that self-insured plans could not be mandated to provide claims data to state APCD systems. See SCOTUSblog, "Gobeille v. Liberty Mutual Insurance Company," https://www.scotusblog.com/case-files/cases/gobeille-v-liberty-mutual-insurance-company/, accessed October 7, 2021. A self-insured or self-funded plan is a type of coverage offered by some employers whereby the employer covers the cost of claims as they accrue rather than paying an insurance premium to an insurance carrier. Such plans that were reporting to the Rhode Island APCD are classified as commercial plans.
} 
2015. ${ }^{18}$ Treatments and medications paid for out of pocket or using non-insurance subsidies are not recorded in the data.

Buprenorphine prescriptions are identified in the pharmacy claims file using National Drug Code (NDC) numbers and are restricted to formulations approved for the treatment of opioid use disorder. The pharmacy payer is also included in the pharmacy claims data. The list of NDC codes for the set of included buprenorphine formulations can be obtained from the authors upon request. The data indicate when a claim was denied by the given insurer, and any such denied claims are excluded from the analysis. We define opioid use disorder as having a diagnosis of either opioid abuse or opioid dependence, based on the ICD-9 codes observed in the medical claims file. ${ }^{19}$ We define a high-dose opioid prescription as one involving a total daily dosage of 90 morphine milligram equivalents (MME) or greater. ${ }^{20}$

The APCD data also contain records pertaining to uninsured individuals (who did not qualify for Medicaid) who accessed federal funds to pay for substance abuse treatment under Rhode Island's global waiver agreement (Rhode Island Executive Office of Health and Human Services 2012), as these funds were administered by the state's Medicaid program. These records are marked using the aid category "costs not otherwise matchable," or "CNOM" for short. Some individuals observed with this aid category are also intermittently or subsequently observed as Medicaid enrollees. However, their health services utilization records appear erratic in the APCD.

\footnotetext{
${ }^{18}$ In fiscal 2012 (July 2011 through June 2012), HealthFacts RI enrollees represented 76 percent of all insured Rhode Island residents; by fiscal 2015 that figure had increased to 88 percent, but by fiscal 2018 it had fallen to 77 percent due to the exit from the sample of enrollees in self-insured plans in fiscal 2016. These estimates are based on average monthly APCD enrollment per fiscal year (for fiscal years 2012 through 2018) as compared with external estimates of the total number of insured Rhode Island residents for the same fiscal years. See Burke et al. (2021) for details.

${ }^{19}$ The ICD-9 refers to the ninth revision of the World Health Organization's (WHO) International Classification of Diseases, an internationally standardized classification system for medical conditions and procedures. The WHO published a 10th revision to the system (ICD-10) in 1994 and an 11th revision (ICD-11) in 2019. However, it is common for WHO member countries to adopt new ICD revisions with a lag. In our data set, consistent with the US policies that prevailed at the time, the diagnosis codes and procedure codes through September 2015 use the ICD-9, and the codes from October 2015 onward use the ICD-10. The ICD-10 includes an additional diagnostic category that is not present in the ICD-9, termed "opioid use," which is folded into our definition of opioid use disorder starting in October 2015. However, the number of relevant sample observations involving a diagnosis of opioid use is negligible. For more information, see the World Health Organization's ICD information page, https://www.who.int/standards/classifications/classification-of-diseases, accessed October 25, 2021, and the United States Centers for Medicare and Medicaid Services ICD-10 information page at https://www.cms.gov/Medicare/Coding/ICD10, accessed October 25, 2021.

${ }^{20}$ Opioid prescriptions and their MME values are identified using the APCD pharmacy claims data together with the 2020 Oral MME Conversion File from the CDC. The list of NDC numbers used to identify opioid prescriptions can be obtained from the authors on request.
} 
Therefore, in the analysis that follows we drop all observations of those who are ever observed with that aid category, except when indicated otherwise in the discussions below.

In a companion paper (Burke et al. 2021), we compare the quarterly number of Medicaid-paid prescriptions for buprenorphine in our APCD data (2021Q1 through 2018Q4) with a comparable measure based on the publicly available Medicaid State Drug Utilization data (SDUD) for Rhode Island. We find that the APCD tracks both the movements and levels of the SDUD very closely. Those results offer reassurance that the APCD data are not subject to large irregularities that would distort the assessment of time trends in Medicaid-paid buprenorphine prescriptions. However, the SDUD counts are not available separately for incumbent and new (post-2014) enrollees.

\section{3b. Geographic Unit of Analysis for Treatment Effects}

The data include patient Zip codes, but these are too small geographically and too numerous (77 in total) to serve as meaningful treatment zones. Instead, we use the county subdivision as the relevant treatment geography. Rhode Island consists of 39 (non-water) county subdivisions corresponding to 31 towns and 8 cities. We mapped Rhode Island Zip Code Tabulation Areas (ZCTAs) into county subdivisions using a 2010 Census crosswalk; ${ }^{21}$ for any given ZCTA, we assigned all residents to the county subdivision that accounted for the largest share of the population as of 2010. In most cases the crosswalk was not problematic. In more than half (44 out of 77) of Rhode Island ZCTAs, 100 percent of the population resided in a single county subdivision. In all but seven ZCTAs, a single county subdivision accounted for more than 90 percent of the population, and in all but two ZCTAs, more than 60 percent resided in a single county subdivision. For each of the remaining two ZCTAs, more than 50 percent resided in a single county subdivision. The ZCTAs with more than 90 percent of the population residing in a single county subdivision represent more than 88 percent of the observations in our analysis sample.

\footnotetext{
${ }^{21}$ ZCTAs, created and trademarked by the U.S. Census Bureau, are "areal representations of United States Postal Service Zip Code Service Areas." ZCTAs are less subject to change over time than Zip codes, as the latter represent collections of mail delivery routes rather than geographic areas. For more information, see https://www.census.gov/programs-surveys/geography/guidance/geo-areas/zctas.html, accessed October 19, 2021.
} 
Assigning the county subdivision as the treatment unit assumes that different cities and towns have at least partly distinct markets for buprenorphine treatment — that individuals tend to visit providers in their same city or town, and providers tend to serve patients in a given city or town or set of towns, rather than being equally likely to serve people in all parts of the state. The APCD data report Zip codes for buprenorphine providers (and for providers in general), but previous research shows that these data are not reliable indicators of where the provider actually dispenses such treatments. Complicating the matter, individual providers often serve patients at multiple locations. The literature suggests that individuals prefer to visit primary care providers within 10 miles or less of their residence (Langabeer et al. 2020). Patients receiving prescription buprenorphine are required to make frequent visits to their provider to receive additional dosestypically daily in the initiation phase and then weekly or at least monthly during the maintenance phase. ${ }^{22}$ Therefore, proximity may play an important role in a patient's selection of a buprenorphine provider even more so than in the selection of a primary care provider, who is visited less often. On the other hand, Rhode Island is a small state, with many cities and towns situated quite close to each other, and most likely not every city or town has an office-based provider who is approved to prescribe buprenorphine. In the extreme, if Rhode Island constitutes a single health-care market (for buprenorphine treatment at least), we would not expect to see differential effects of ACA implementation across different cities and towns even if the policy had causal effects in the aggregate.

As described in Section 4 below, as a proxy for intensity of treatment under the ACA at the county subdivision level, we construct health uninsurance rates by county subdivision using data from the American Community Survey (ACS). These rates pertain to the population aged 18 to 64 and represent five-year estimates covering the 2009-2013 period, as single-year estimates are not available for Rhode Island county subdivisions. We use the 18-64 age range, because those over 65 were not targeted by the ACA, and we don't use health outcomes in the APCD for those under age 18. The ACS data's five-year estimates by county subdivision are reported as numbers of uninsured individuals aged 18 to 34 and number of uninsured aged 35 to 64 . The same data source furnishes five-year estimates of population size by county subdivision for those same age

\footnotetext{
${ }^{22}$ See the manufacturer's prescribing information for Suboxone, a brand-name formulation of buprenorphine (combined with naloxone) that is used to treat OUD: https://www.suboxone.com/pdfs/prescribing-information.pdf. See also Clinical Guidelines for the Use of Buprenorphine in the Treatment of Opioid Addiction, SAMHSA, 2004.
} 
ranges. To calculate the uninsured rate for the population aged 18 to 64 , we add the numbers of uninsured in the two smaller age ranges and divide by the sum of the population sizes in those two age ranges.

\section{3c. Analysis sample construction}

Although the APCD data include observations from as early as April 2011 and as late as May 2019, our analysis of buprenorphine receipt among Medicaid incumbents is restricted to the period from January 2012 through December 2015. We chose this time window in order to balance the desire to trace the impact of the ACA over an extended time period-which is important if any effects set in with a delay_against the goal of maintaining a relatively balanced and sufficiently large panel of incumbent enrollees. The panel of Medicaid incumbents would be subject to increasing attrition after 2015, and furthermore, the APCD's estimates of nonMedicaid enrollment growth would become less accurate starting in 2016 due to the omission of enrollees in self-insured plans (for reasons described in Section 3a. above). A separate issue favoring the selected time window is that, starting in July and August of 2016, new federal policies were implemented that expanded the pool of potential buprenorphine prescribers and raised patient limits for experienced prescribers. ${ }^{23}$ We want to avoid any influence of these policies when measuring the impact of the ACA on prescriber stocks and prescribers' patient loads in Rhode Island.

To construct our analysis sample — called the "Medicaid incumbent sample" or "incumbent sample" for short - we first remove all observations of anyone ever observed with the CNOM aid category code (described in Section 3a above). Individuals with inconsistent age changes or gender changes over time as well as individuals who enrolled exclusively in pharmacy insurance plans (with no observed medical insurance) are also excluded from the sample. We drop all observations from the county subdivisions representing Block Island and Central Falls, as each

\footnotetext{
${ }^{23}$ For information about these policy changes, visit, for example, https://nasadad.org/2019/01/bupecap/ and https://www.asam.org/advocacy/practice-resources/buprenorphine-waiver-management, both accessed on October 7 , 2021. For discussions of how these policy changes may have influenced buprenorphine prescribing, see Burke et al. (2020), Burke et al. (2021), and Karen E. Johnson et al., "The Effects Of The Comprehensive Addiction and Recovery Act of 2016 on Nurse Practitioner and Physician Assistant Buprenorphine Prescribing in Medicaid," Health Affairs blog, November 6, 2019, https://www.healthaffairs.org/do/10.1377/hblog20191105.242580/full/.
} 
of these places has an extreme value for at least one of the key variables used in the regression analysis (discussed below), and Block Island consistently has very small sample sizes.

Following the various exclusions, we select all Rhode Island residents aged 19 and older (as of January 2013) who were enrolled exclusively in Medicaid in all of their available observations dated from January 2012 through December 2014, and who were observed in at least 22 of the 24 months dated January 2013 through December 2014. For the selected set of individuals, we then use all available observations spanning the period from January 2012 through December 2015 (for reasons given just above in this same section), provided the individual was enrolled in Medicaid insurance in the given month. Medicaid enrollees in our definition do not include those dually eligible for Medicaid and Medicare. ${ }^{24}$ The resulting sample consists of 1,503,760 observations representing 32,654 unique individuals. This means that the average person in the sample has 46 observations_-indeed, more than half of sample members are observed in all 48 months of the time period.

\section{3d. Estimating Buprenorphine Provider Supply and Average Patient Loads}

To estimate the supply of buprenorphine prescribers and the average patient load per prescriber over the relevant time period, we use the pharmacy claims data, which indicate the prescribing provider associated with each prescription. For these purposes we use the pharmacy claims for an unrestricted enrollee sample rather than restricting to the incumbent analysis sample. ${ }^{25}$ A practitioner is said to enter as a buprenorphine prescriber in the first month in which they appear as the prescribing provider in a non-denied claim for an admissible formulation of buprenorphine (or "valid" claim). A prescriber is active in a month if they appeared in at least one valid claim in the month. A practitioner is said to exit as a buprenorphine prescriber if they have a non-prescribing or inactive spell of at least six consecutive months that is never followed by an active spell of any length, and the exit month is the first month of such an inactive spell. ${ }^{26} \mathrm{We}$ define entry into, activity in, and exit from Medicaid prescribing similarly, but using only the claims for

\footnotetext{
${ }^{24}$ Such "dual eligibles" would have qualified for Medicare based on age or disability and also qualified for either full or partial Medicaid benefits based on their income. Medicare represents their primary insurance, and the Medicaid plan is used to pay for services covered only by Medicaid and/or to pay for out-of-pocket expenses not covered by Medicare.

${ }^{25}$ We require that the enrollee on each claim be at least 18 years of age and have a Rhode Island Zip code. The prescriber must also report a Rhode Island Zip code.

${ }^{26}$ In identifying entry and exit, to avoid left- and right-censoring we make use of data that start in 2011Q2 and extend through $2017 \mathrm{Q} 4$.
} 
buprenorphine prescriptions of Medicaid enrollees. Given these definitions, prescribers per month is simply the number who have entered but not yet exited as of that month, regardless of activity in the given month, and Medicaid prescribers per month is analogous. Average patients per prescriber is calculated as the average across providers of the number of unique buprenorphine patients per month associated with that prescriber. Some patients filled prescriptions from more than one prescriber in a single month, and this overlap is not eliminated from the calculations.

Although this approach will miss any authorized or "DATA-waived" practitioners ${ }^{27}$ who prescribed exclusively to individuals not observed in the APCD or who were simply never active, for 2014 and 2015 our prescriber counts are nearly identical to the counts of official DATA-waived practitioners in Rhode Island as furnished to us by the Rhode Island Department of Health. We have so far been unable to obtain a source for the number of DATA-waived practitioners in Rhode Island for 2012 and 2013.

\section{3e. Incumbent Sample Properties and Overall Enrollment Trends}

We begin by illustrating the size of the incumbent sample over the time period of interest, alongside other measures that show the growth in health insurance enrollment observed in the Rhode Island APCD in and after January 2014. As seen in Figure 1, the incumbent sample size is quite stable over time, despite (based on how it was constructed) being somewhat smaller in 2012 and 2015 compared with the 2013-2014 interval. The figure also shows enrollment per month (by Medicaid status) within a "basic" sample requiring only that an enrollee have six consecutive observations in the APCD from April 2011 through May 2019 (after imposing the other exclusions described above, such as residing in Rhode Island and not exhibiting irregular age or gender changes). Prior to January 2014, the number of Medicaid enrollees in the basic sample in any given month is roughly twice as large as the number in the Medicaid incumbent analysis sample. However, in 2014, average monthly Medicaid enrollment in the basic sample surges by 71 percent from its 2013 average level, with most of those gains occurring in the first

\footnotetext{
${ }^{27}$ Under the Drug Addiction Treatment Act of 2000 (DATA), qualified practitioners can become authorized to prescribe buprenorphine by successfully completing the requirements for a so-called X waiver from the U.S. Drug Enforcement Administration (DEA). For more information, see University of Pennsylvania's Center for Addiction Medicine and Policy, https://penncamp.org/education/what-is-an-x-waiver-and-who-needs-one/, accessed October 19, 2021.
} 
three months of 2014. Average monthly non-Medicaid enrollment also exhibits a significant increase in 2014 (from 2013), albeit at the much lower rate of 7.4 percent, and the gains are concentrated in January 2014. Medicaid's share in the basic sample increases substantially over the time period, from 11 percent of enrollees on average in the pre-2014 period to more than 17 percent in the 2014-2015 period.

Based on extensive previous research for the United States as whole, we take it as given that enrollment increases of these magnitudes would not have occurred absent the ACA. ${ }^{28} \mathrm{We}$ do present limited evidence (Section 5) in support of this proposition for Rhode Island specifically, but it is beyond the scope of this paper to identify the quantitative effect of the ACA on health insurance enrollment in the state.

As shown in Table 1, the Medicaid incumbent analysis sample differs significantly along a few relevant dimensions from the Medicaid subset of the basic sample, comparing the sets of individuals in those respective samples over the 2012-2013 (pre-ACA) time period. ${ }^{29}$ In particular, members of the Medicaid incumbent analysis sample were significantly more likely to have filled at least one buprenorphine prescription compared with members of the basic sample's Medicaid subset (2.8 percent versus 1.8 percent). Analysis sample members were also more likely to have been diagnosed with a substance use disorder, with opioid use disorder in particular, with alcohol use disorder in particular, and more likely to have ever received a highdose opioid prescription. While both samples are heavily female - as was typical of Medicaid enrollment prior to the expansion - the analysis sample shows an even larger female share than the basic sample, and a slightly higher average (median) age. It is to our advantage that the analysis sample overrepresents buprenorphine recipients, as the regression analysis will be less susceptible to problems associated with rare outcomes, especially since the models include county subdivision fixed effects. Furthermore, the fact that the analysis sample is strongly balanced means that the results will be less subject to biases related to observing some individuals only intermittently. Nonetheless, the results derived from the analysis sample may

\footnotetext{
${ }^{28}$ See, for example, Courtemanche et al. (2017); Simon, Soni, and Cawley (2017); Miller and Wherry (2017); Lipton, Decker, and Sommers (2017); Kaestner et al. (2017); Gangopadhyaya and Johnston (2021); and Antwi, Moriya, and Simon (2015).

${ }^{29}$ The Medicaid subset of the basic sample, used to calculate the values in column 2 of Table 1, consists of members of the basic sample (described above) who were enrolled in Medicaid in at least three months during the period from January 2012 through December 2013.
} 
not be indicative of patterns in buprenorphine utilization over time in the less-restricted sample of Medicaid enrollees.

Figure 2 shows the monthly rate of buprenorphine utilization from January 2012 through December 2015 for the analysis sample, measured as the number of individuals who filled at least one buprenorphine prescription in the month (per 100,000 enrollees). The figure also includes a dashed gray line showing the predicted number of buprenorphine recipients per month (per 100,000 enrollees), based on the fitted linear trend in buprenorphine receipt for the 20122013 period. ${ }^{30}$

The predictions for the 2014-2015 period represent naïve estimates of the counterfactual outcomes that would have emerged among our incumbents had Rhode Island not implemented the ACA and Medicaid expansion in January 2014. As the realized rates of buprenorphine receipt in the 2014-2015 period fall short of the predicted values — and by an increasing margin on average over time - the comparison shows that among incumbents, the pace of the increase in buprenorphine-prescription receipt slowed in the latter (post-ACA) period relative to the initial period. Additional analysis shows that this pattern does not reflect any increase in the supply of buprenorphine pills per prescription that would have enabled patients to fill prescriptions less frequently. However, the true counterfactual outcomes for this sample for the 2014-2015 period are unknown. Therefore, based on this evidence alone we can't conclude that the slower growth in buprenorphine receipt among Medicaid incumbents in the 2014-2015 period was actually caused by the ACA. For example, the underlying demand for buprenorphine within a relatively fixed group of individuals simply may have been expected to plateau after a certain amount of time.

\section{Testing for Causal Effects of ACA Implementation on Buprenorphine Receipt among}

\section{Incumbents: Empirical Models and Identification Strategies}

To assess the causal impact of the ACA on buprenorphine receipt among Medicaid incumbents in Rhode Island, we conduct logistic regression analysis that exploits geographic variation in the

\footnotetext{
${ }^{30}$ The trend is obtained via a linear regression of the monthly values for 2012 through 2013 against the number of months since January 2012 (and a constant), and then by predicting out-of-sample for the months in 2014 through 2015. The qualitative patterns are similar if we estimate the counterfactual trend in the raw number of patients expected to receive buprenorphine per month.
} 
intensity of treatment under the ACA. More precisely, we adopt a difference-in-differences (DID) identification strategy that tests for whether incumbent Medicaid enrollees living in cities and towns that were more intensively treated under the ACA — as measured by local influxes of new health insurance enrollees or proxies for such inflows - experienced differential changes following ACA implementation in their chances of receiving buprenorphine.

The baseline regression model is as follows:

$$
\begin{aligned}
& \operatorname{Logit}\left(p_{i c t}\right)=\alpha_{0} \\
& +\alpha_{1} \text { NonMedicaid_Growth }{ }_{c t}+\alpha_{2} \text { Medicaid_Growth } c t \\
& +\alpha_{3}\left(\text { NonMedicaid_Growth } h_{c t} * \text { Post_2014t }\right)+\alpha_{4}(\text { Medicaid_Growth } c t \\
& \text { * Post_2014t })+\alpha_{5} X_{i c t}+\mu_{c}+\tau_{t} \\
& p_{i c t}=P\left(y_{i c t}=1 \mid \text { NonMedicaid_Growth }{ }_{c t}, \text { Medicaid_Growth } h_{c t}, \text { Post_2014 }{ }_{t}, X_{i c t}, \mu_{c}, \tau_{t}\right)
\end{aligned}
$$

In the first equation above, the dependent variable is the logit transformation (log odds) of the probability $\left(p_{i c t}\right)$ of filling a buprenorphine prescription in a given month for individual $i$ residing in county subdivision $c$ as of month $t$, based on the observed binary dependent variable, $y_{i c t}$, of actual buprenorphine receipt. NonMedicaid_Growth $h_{c t}$ refers to our measure of (gross) yearly growth in non-Medicaid enrollment, which is calculated by dividing the number of new nonMedicaid enrollees in county subdivision $c$ in year $t$ by the total number of (unique) non-Medicaid enrollees in the same county subdivision in year $t-1 .{ }^{31}$ This measure differs from the net percentage change in non-Medicaid enrollment by county subdivision over the equivalent time period, because (1) the numerator includes only non-Medicaid enrollees who never appeared in the APCD sample previously with any type of insurance, and (2) the denominator doesn't exclude those who exit non-Medicaid enrollment between year $t-1$ and year $t$. This approach deliberately isolates the contribution of first-time enrollment in a year, which starting in 2014 is likely to capture those

\footnotetext{
${ }^{31}$ In order to calculate (Medicaid or non-Medicaid) enrollment growth in 2012 (from 2011) we need measures of the "full-year" enrollment by county subdivision for 2011 - that is, the total number of enrollees observed in at least one month of that year in the given place. However, for 2011 we observe enrollment only for April through December, and so we may miss some enrollees. To impute full-year enrollment by county subdivision for 2011, we make use of the enrollment data from the years 2012 and 2013, for which we observe all 12 months. For each county subdivision as of 2012, we calculate the ratio of full-year enrollment to April-December enrollment and then take the average ratio across all county subdivisions. We do the same for 2013 and then take the average of those two results. The resulting value is used as a multiplication factor to scale up our April-December enrollment numbers for 2011 to full-year 2011 enrollment (by county subdivision). We do this procedure separately for Medicaid enrollment and non-Medicaid enrollment.
} 
newly eligible for health insurance under the ACA. The term Medicaid_Growth ct $_{\text {refers to (gross) }}$ yearly growth in Medicaid enrollment, which is measured analogously but using first-time Medicaid enrollees in a place and year as a share of Medicaid enrollees in the same place in the preceding year. In both cases, we use an unrestricted sample to construct these treatment measures, not the analysis sample, as the analysis sample does not capture anyone who first enrolled in 2014 or later. ${ }^{32}$

The dummy variable Post_2014 refers to whether the observation was dated in either 2014 or 2015 or instead in either 2012 or 2013. The latter terms appear only in interactions with the enrollment growth terms, as aggregate time effects, denoted by $\tau_{t}$, are captured by including monthly dummy variables (omitting January 2012). Fixed effects on buprenorphine receipt by county subdivision are denoted by $\mu_{c}$, and these are captured as the coefficients on county subdivision dummies (omitting Providence, which accounts for the largest share of observations). For this geographic influence, we have in mind supply-side factors such as the number of buprenorphine prescribers with offices in or close to the subdivision, the number of specialty treatment facilities offering methadone (a potential substitute for buprenorphine), and any common demand-side factors within the county subdivision not captured in the individual characteristics.

The term $X_{i c t}$ refers to a vector of individual-level variables (and its coefficient, $\alpha_{5}$, also represents a vector). In the baseline model, these include gender, age range as of the first available observation in 2013, and indicators of ever having had (in any of their observations in the data set) each of the following health outcomes: diagnosis of alcohol use disorder, diagnosis of a substance use disorder other than OUD, diagnosis of opioid overdose, and receipt of a high-dose opioid prescription. ${ }^{33}$ As these are all time-invariant factors, they aim to capture the individual's idiosyncratic demand for buprenorphine on average over the time period, in lieu of fixed individual effects. A time-varying idiosyncratic error term is suppressed from the logit equation, as the residual refers to the

\footnotetext{
${ }^{32}$ For 2012 only, the enrollment growth measures count any members of our incumbent sample who were first-time insurance enrollees in 2012 (not observed in 2011). This may result in a modest correlation between sample sizes and enrollment growth by county subdivision in the analysis sample in 2012, but it is not expected to introduce any significant biases in the coefficient estimates.

${ }^{33} \mathrm{~A}$ high-dose opioid prescription is defined as one involving a daily dosage of 90 morphine milligram equivalents (MME) or higher. This is calculated using information from the Rhode Island APCD's pharmacy claims file and the CDC's 2020 Oral MME Conversion File. See https://www.cdc.gov/opioids/data-resources/index.html.
} 
difference between a given realized (binary) outcome and the fitted probability - a residual probability can't be estimated at the individual level.

Note that the model doesn't include an indicator of whether the individual was ever diagnosed with opioid use disorder over the time period. Although the presence of opioid use disorder is in principle a strong predictor, or even a necessary precursor, of demand for buprenorphine, an OUD diagnosis in our data should be viewed as an endogenous outcome, as it also requires an office visit or hospital stay in which the diagnosis is recorded. Very often in our data, an individual's first OUD diagnosis coincides very closely with his or her first buprenorphine prescription - the two events often occur in the same month or even on the same day, and it is not uncommon for the treatment to precede the diagnosis. These facts suggest that both the OUD diagnosis and the buprenorphine prescription are precipitated by a common unobserved factor, rather than the diagnosis being an exogenous precursor to treatment. While the model's included diagnosis variables (such as alcohol use disorder diagnosis) can also be viewed as endogenous outcomes, in the data they are less simultaneous with buprenorphine receipt than is the OUD diagnosis. The conditional logit model discussed below resolves these concerns by controlling for all fixed predictors of buprenorphine receipt per individual, both observed and unobserved, but it has other limitations that are discussed below.

The motivation for the empirical model is as follows. Being subject to an influx of new enrollees (represented by Medicaid_Growth ${ }_{c t}$ or NonMedicaid_Growth ${ }_{c t}$ ) in one's local area could impede access to buprenorphine among Medicaid incumbents if it increases demand for the treatment without an adequate supply response, as any individual buprenorphine provider is subject to both legal and practical limits on their ability to take on new patients. New Medicaid enrollees might be more of a problem than other new enrollees, as they would compete for the same limited set of providers that accept Medicaid patients. Alternatively, an enrollment increase could have a neutral or even a beneficial effect on buprenorphine access for Medicaid incumbents if it induces more providers to serve that market. Any of these effects might have occurred in response to enrollment increases even before enactment of the ACA, and these are captured in the estimation model above as the standalone coefficients on the enrollment growth variables. However, enrollment increases owing to the ACA specifically might have had a different impact than previous enrollment increases, for a few reasons: (1) individuals who gained access to non- 
Medicaid health insurance under the ACA are more likely to have gained coverage for substance abuse treatment than those who entered non-Medicaid plans prior to 2014 owing to the new mental health parity and essential health benefits requirements of the ACA, ${ }^{34}$ and (2) people who became eligible for Medicaid under the ACA's expanded eligibility rules would have differed from those who joined Medicaid in earlier years (for example, poor childless adults became newly eligible). For both of those reasons, post-2014 enrollees may have been more likely to demand buprenorphine upon enrolling than those who first enrolled in 2012 or 2013, leading to more intense spillover effects onto incumbents in the post-ACA period. Separately, the larger scale of the enrollment increases under the ACA (observed in 2014 in particular) might have induced a greater supply response than did earlier (smaller) enrollment increases, again implying stronger benefits for incumbents in the post-ACA era than previously.

Accordingly, the causal effects of the ACA are estimated via the coefficients, $\alpha_{3}$ and $\alpha_{4}$, on the respective interaction terms (in equation 1 above) between the Post_2014 dummy and the nonMedicaid and Medicaid enrollment increase variables. These interaction coefficients capture any differential change (in the post-2014 period compared with the earlier period) in the chance of receiving buprenorphine owing to differences across county subdivisions in the intensity of treatment with new enrollees in the post-ACA period. Based on the mechanisms just discussed, we would expect negative interaction coefficients if demand-side competition or capacityconstraint effects were dominant, and positive interaction coefficients if supply-side responses were dominant. Because the model includes county subdivision dummies and controls for any pre-existing relationship (before 2014) between annual (Medicaid and non-Medicaid) enrollment growth and outcomes, it represents a conservative test of the ACA's causal effects. In order to obtain a significant coefficient on the interaction between the Post_2014 dummy and either of the treatment variables, it's necessary that (a) the relationship between enrollment growth and outcomes (within county subdivisions) changed significantly in the post-2014 period compared with the pre-2014 period, and (b) enough county subdivisions experienced atypical enrollment

\footnotetext{
${ }^{34}$ This claim is supported by evidence presented by Wen et al. (2013), who examines the impact of state mentalhealth parity laws (implemented prior to the ACA) on access to mental health services.
} 
growth rates in 2014 and/or 2015 (relative to the prior experience in each place) such that enrollment growth effects are not absorbed in the county subdivision fixed effects. ${ }^{35}$

The basic identifying assumption is that there are no possible causes for observing significant coefficients on the interaction terms other than the causal mechanisms related to the ACA described above. That is, absent those mechanisms buprenorphine receipt among incumbents would not have otherwise experienced differential changes in the post-2014 period that aligned with their respective enrollment growth rates by county subdivision in the post-2014 period, netting out the other factors accounted for in the model.

To consider just one possible violation of this assumption, we might obtain a spurious positive coefficient on the Medicaid_Growth ${ }_{c t} *$ Post_2014 $_{t}$ term if some county subdivisions were experiencing relatively fast acceleration in buprenorphine demand in their low-income (or Medicaid-targeted) populations in 2014 from the previous year, resulting in both greater increases in buprenorphine receipt among Medicaid incumbents after 2014 (compared with other county subdivisions) and greater increases in Medicaid enrollment (compared with other county subdivisions and their own history) as more individuals sought first-time Medicaid coverage for buprenorphine treatment. A similar relationship might apply to non-Medicaid enrollment growth, as under the ACA, many non-Medicaid enrollees were also of low income. That is, the identification is vulnerable to the possibility that the county subdivisions responding most to the ACA and Medicaid expansion (in terms of enrollment growth) are the same ones that were poised to experience either faster or slower growth in buprenorphine utilization among incumbents after 2014 due to underlying local trends in the demand for and/or supply of buprenorphine. ${ }^{36}$

\section{4b. Alternative model}

Given the potential endogeneity of the observed enrollment increases as measures of treatment intensity, we estimate an alternative to the baseline model that uses pre-ACA uninsurance rates

\footnotetext{
${ }^{35}$ Less conservatively, we might infer causal effects of the ACA based on observing (a) any significant relationship between enrollment growth and outcomes over the entire period, even if unchanged after 2014, and (b) excess enrollment growth rates in 2014 and/or 2015 that are attributable to the ACA. The impact of that excess enrollment growth on outcomes could then be attributed to the ACA.

${ }^{36}$ However, this spurious relationship could be prevalent regardless of whether the increases in Medicaid enrollment were caused by the Medicaid expansion or instead would have occurred even without the expansion.
} 
by county subdivision observed in the American Community Survey. As described in Section 2 above, the rates we use for Rhode Island county subdivisions pertain to the population aged 18 to 64 and represent five-year estimates covering the 2009-2013 period, as single-year estimates are not available for Rhode Island county subdivisions. At least three previous papers utilize prepolicy uninsurance rates as measures of treatment intensity under various health insurance reform policies (Finkelstein 2007, Miller 2012, and Courtemanche et al. 2017), the idea being that local areas that had greater shares of uninsured individuals prior to a given health insurance expansion would have had a greater intended treatment intensity. The findings of Courtemanche et al. (2017) in fact show that actual enrollment increases align well with the pre-policy uninsurance rates by core-based statistical area (CBSA). The uninsurance rate has the advantage of being less subject to the endogeneity concerns discussed above regarding the realized enrollment increases, as the former are measured prior to the policy intervention. However, a drawback of the uninsurance rate, in the case of our data at least, is that although it represents a strong predictor of non-Medicaid enrollment growth under the ACA, it is not significantly associated with Medicaid enrollment growth—see Appendix Figure A1. Therefore, this alternative model can test only for the causal effects of non-Medicaid enrollment increases under the ACA on buprenorphine utilization among incumbents. The model is as follows:

$$
\operatorname{Logit}\left(p_{i c t}\right)=\alpha_{0}+\alpha_{1}\left(\text { Uninsured }_{c, 2013} * \text { Post_2014t }_{t}\right)+\alpha_{5} X_{i c t}+\mu_{c}+\tau_{t}
$$

In the above, the term Uninsured $_{c, 2013}$ refers to the uninsurance rate in county subdivision $c$ as of the 2009-2013 five-year estimate, considering individuals aged 18 to 64 in the subdivision. Notice that this alternative model does not contain the stand-alone uninsurance rate as a separate term. As those rates (by construction) don't vary within county subdivisions over time in our data, the stand-alone term would be collinear with the county subdivision dummies. In this model the causal effects of the ACA (considering any spillover effects of non-Medicaid enrollment increases) are captured by the coefficient $\alpha_{1}$. That is, the model tests whether individuals living in county subdivisions that had different pre-ACA uninsurance rates (on average over the 2009-2013 period) experienced significantly different changes in their chances of receiving buprenorphine in the post-2014 period compared with the 2012-2013 period. Identification rests on the assumption that the pre-ACA uninsurance rates are not correlated with unobserved factors that would have predicted county-subdivision trends in buprenorphine rates 
that were to emerge in the 2014-2015 period. A possible violation is that places with high uninsured rates were relatively poor and so may have been poised to experience sharper increases in OUD prevalence and therefore greater increases in demand for buprenorphine among incumbent enrollees during the 2014-2015 period - this would result in a spurious positive coefficient on the relevant interaction term above.

\section{4c. Conditional Logit Model}

The models described so far control only for observed, time-invariant characteristics at the individual level, a feature that may limit the models' explanatory power and could contribute to omitted-variable biases in the coefficient estimates. We also estimate a conditional logit model that controls for fixed individual heterogeneity and introduces time-varying factors at the individual level. The "ever" diagnosis variables must be dropped (because they are timeinvariant within each individual), and we include indicators of whether in the given month the individual (a) received a high-dose opioid prescription and (b) was diagnosed with a nonfatal opioid overdose. The model does not estimate fixed effects for each individual but instead conditions the marginal effects on the total number of instances of buprenorphine receipt per individual. Population average marginal effects cannot be derived. County subdivision dummies and monthly dummies are directly estimated, as previously. As in the alternative model just described, for the treatment measure we use the uninsured rate by county subdivision from 2009 through 2013 and test for causal effects of the ACA based on the interaction of that term with the Post_2014 dummy.

The sample can include only those individuals who received buprenorphine at least once among their observations but not in all of their observations for the time period. We are left with roughly 54,600 observations pertaining to 1,168 unique individuals, each of whom is observed an average of 46.7 months. The identification of the county subdivision effects requires that sufficient numbers of individuals move across different county subdivisions over the time period. Two county subdivisions are dropped from the estimation because either all of their observations involve buprenorphine receipt or none does.

\section{Results}


Before describing the regression results, we consider some basic descriptive statistics of the analysis sample for each year from 2012 through 2015, shown in Table 2. Each value represents the mean of a given variable over all observations in the analysis sample dated within the given calendar year. (Enrollment increases and uninsured rates are presented as percentages, and all other values are given as proportions.) Not surprisingly the average enrollment increaseswhether non-Medicaid or Medicaid - are greatest in 2014, the year in which the ACA was implemented in Rhode Island. Although the average enrollment growth rate for either type of insurance is lower in 2015 alone than it is on average in 2012 through 2013, when the two-year post-ACA period of 2014 and 2015 is considered, the average enrollment increase exceeds that of the pre-ACA period. The share of monthly observations in a given year in which an individual filled a buprenorphine prescription (the dependent variable) increases in each year of the time period, consistent with the pattern in Figure 2. All remaining variables described are factors that are constant within an individual over time or (in the case of uninsurance rates) constant within a county subdivision over time. As a result, the yearly sample mean values experience very little variation over time, and such variation owes only to the small changes in sample composition that occur from month to month.

Given these patterns, in the baseline model the time variation in buprenorphine utilization is expected to load primarily onto the monthly dummies, the enrollment-increase variables (which vary by county subdivision and year), and the interaction between the Post_2014 dummy and the enrollment-increase variables. In the alternative model, the time variation will load onto the interaction between the Post_2014 dummy and the percentage of uninsured by county subdivision, in addition to the monthly dummies.

Table 3 shows the coefficient estimates and associated information from the baseline logit model described above. Standard errors are clustered at the level of the individual enrollee. Coefficients on the county subdivision and monthly dummies are suppressed from the table, but each of those sets of factors is jointly significant in the regression. The table shows the raw (unexponentiated) coefficients. Negative coefficients indicate that a factor is associated with a lower chance of receiving buprenorphine, and positive coefficients indicate the opposite. The enrollment growth variables have been recentered around their sample means, so that the coefficient estimates shown are evaluated at those means. 
The stand-alone coefficients on non-Medicaid and Medicaid enrollment growth measures are in both cases negative but statistically insignificant - these reflect the relationship between enrollment growth (by county subdivision and year) in the 2012-2013 period. The estimated interaction effects are also statistically insignificant for either type of enrollment growth, but the point estimates are positive in both cases. (At the bottom of the table, the net effect of enrollment growth in the 2014-2015 period is calculated by taking the sum of the stand-alone and interaction coefficients just described. Neither effect is significant.) The results indicate that, even allowing for the possibility that enrollment growth was negatively associated with buprenorphine utilization prior to ACA implementation (based on the sign of the point estimates), that association was not significantly different following the introduction of the ACA, and if anything, it became less negative. In sum, the results do not offer any support for the claim that surges in enrollment growth under the ACA would have made it harder for incumbents to fill buprenorphine prescriptions. Furthermore, based on these results, we can't reject the proposition that the association between enrollment growth (of either type) and buprenorphine utilization was null throughout the time period.

Individual factors that have significant positive associations with buprenorphine receipt include ever being diagnosed with alcohol use disorder, ever having a non-OUD substance use disorder diagnosis, a nonfatal opioid overdose (ever), and being age 29 or older (as opposed to being aged 19 to 28) as of 2013. Individuals who ever received a high-dose opioid prescription were significantly less likely to receive buprenorphine prescriptions compared with those who never had such a prescription, conditional on the other factors.

The model is highly jointly significant and yields a pseudo R-squared value of 0.173 , suggesting somewhat limited explanatory power. Figure 3 shows the predicted and actual shares of buprenorphine recipients per month for each of four county subdivisions. The model offers a relatively good fit in the case of Providence, which accounts for more observations than any other county subdivision, as well as for Cranston, another relatively populous city. The fit is weaker in the case of Warwick and Johnston, both of which exhibit relatively high buprenorphine utilization relative to their sample sizes. These illustrations show that some places saw monthly movement in buprenorphine receipt that deviated from the aggregate month effects not captured by changes in their enrollment growth rates, which by construction varied only at 
the annual frequency. As indicated in the figure, the different locations varied in terms of their 2014 enrollment growth rates.

Figure 4 compares the predicted change in the chance of receiving buprenorphine before versus after 2014 by county subdivision; the subdivisions are ordered according to their respective nonMedicaid enrollment increases in 2014 (left panel) or according to their Medicaid enrollment increases in 2014 (right panel). The predicted rates are evaluated at the actual values of covariates in each county subdivision - these are not "ceteris paribus" comparisons that isolate any particular marginal effect. In the left panel, there appears to be a modest positive association between the predicted increase in buprenorphine receipt and the 2014 increase in non-Medicaid enrollment — the height of the bars is increasing from left to right—although this relationship is dominated by Providence and derives from the imprecise interaction coefficient described above. The right panel shows that there is no systematic pattern in the predicted changes when the county subdivisions are ordered according to their 2014 Medicaid enrollment increases, also consistent with the results from Table 3 .

Table 4 shows the coefficient estimates from the alternative model that employs the pre-2014 uninsurance rates by county subdivision as the indicator of treatment intensity. (Standard errors are again clustered by individual enrollee.) The coefficient on the interaction between the uninsurance rate and the post-2014 dummy is positive and statistically significant, indicating that cities or towns that had higher uninsurance rates - and therefore would have been more susceptible to treatment under the ACA—saw greater estimated increases in buprenorphine utilization after implementation of the ACA. As these were places that, on average, had larger non-Medicaid enrollment increases (and larger overall increases) but not larger Medicaid enrollment increases, the result indicates that non-Medicaid enrollment influxes in 2014 and 2015 did not impose negative effects on incumbents' chances of receiving buprenorphine, and if anything, they contributed to greater gains in utilization in those places following the ACA. Otherwise, the fit of the models is remarkably similar, which indicates that most of the variation is accounted for by the fixed individual factors, the county subdivision effects, and the monthly effects, and very little is accounted for by the treatment variables. The similarity of the fit is seen in Figure 5, which is the equivalent of Figure 3 but uses the alternative model instead of the baseline model. 
Results of the conditional logit model are shown in Table 5. Standard errors are robust but cannot be clustered by individual in this setting. The estimation yields a positive and highly significant coefficient on the interaction between the pre-ACA uninsured rate and the Post_2014 dummy, again suggesting that individuals residing in places that were subject to more intensive treatment under the ACA (in the form of non-Medicaid enrollment growth in 2014 in particular) experienced larger gains in their chances of receiving buprenorphine in a given month, controlling for each individual's fixed tendency to receive buprenorphine in a given month and netting out the influence of the time-varying individual health indicators as well as fixed monthly and county subdivision effects. The larger magnitude of this interaction coefficient compared with the one shown in Table 4 agrees with a prior that the selected individuals in the current estimation should have been more susceptible to any causal effects of the ACA on buprenorphine utilization than the average individual, who most likely has never taken the drug.

The coefficients on the time-varying factors suggest that a given individual is less likely to fill a buprenorphine prescription in a month in which they either (a) received a high-dose prescription

of an opioid medication or (b) experienced a nonfatal opioid overdose. Although these effects are large (especially the opioid prescription effect) and highly significant, either one might be interpreted in a reverse causal sense or may reflect a spurious association based on other unobserved factors.

\section{5b. Pre-ACA Trends in Buprenorphine Use}

The difference-in-differences identification strategy requires that the time trend in buprenorphine use in the pre-treatment period did not differ systematically according to the eventual intensity of treatment. (That condition is necessary but not sufficient for identification of causal effects.) To test this requirement, we identify the (weighted) quartiles of each treatment variable - based on the distribution of each variable in the analysis sample observations from 2014 only — and assign the appropriate quartile indicator to each observation. (These indicators are fixed within a county subdivision across all time periods.) We then run logit regressions on the 2012-2013 subset of the analysis sample in order to test for differences in linear (monthly) time trends in buprenorphine use associated with the eventual intensity of treatment under the ACA, based on the quartile of a given treatment variable. 
As seen in Appendix Figures A2 through A4 and Table A1, we find that most groups exhibited similar trends in buprenorphine utilization in the pre-ACA period. However, there are a few exceptions, as follows: the average growth rate per month in buprenorphine use from January 2012 through December 2013 was significantly lower in county subdivisions with either (a) preACA uninsurance rates in the first quartile of the distribution rather than higher pre-ACA uninsurance rates; (b) non-Medicaid enrollment growth rates as of 2014 in the first quartile of the distribution rather than higher non-Medicaid enrollment growth rates in 2014; or (c) Medicaid enrollment growth rates as of 2014 in the second quartile of the distribution rather than either higher or lower Medicaid enrollment growth rates in 2014. (Those results apply after we control for patient characteristics at the county subdivision level that would predict buprenorphine demand.) In sum, the results suggest that individuals residing in places that were (eventually) subject to less intensive treatment under the ACA also exhibited somewhat slower growth in buprenorphine use in the pre-ACA period.

In light of these results, we conduct a sensitivity analysis of the above regression models in which observations from the "non-conforming" quartile (for a single treatment variable at a time) are omitted from the relevant regression. As seen in Appendix Table A2, columns 1 and 2, the baseline model results (from Table 3 above) are highly robust to dropping observations associated with either the first quartile of 2014 non-Medicaid enrollment growth or the second quartile of 2014 Medicaid enrollment growth. (Results in Table A2 are limited to the interaction coefficients of interest. All other coefficients are suppressed.) However, results of the alternative model are not robust: The coefficient on the interaction between the pre-ACA uninsured share and the Post_2014 dummy is smaller (albeit still positive) and no longer statistically significant. A similar lack of robustness applies to the conditional logit model. See columns 3 and 4 of Table A2. These robust models indicate that the ACA had no significant effects in either direction on incumbents' likelihood of receiving buprenorphine.

\section{Changes in the Supply of Buprenorphine Prescribers and Patient Loads per Prescriber}

The methods of estimating the number of buprenorphine providers per month and the average monthly patient load per provider are described in Section 3d above. These are not pure supplyside measures, as they miss providers who are approved to prescribe buprenorphine but who never match with patients in our sample. Recall that the sample used to calculate these values is 
no longer the incumbent analysis sample but pertains to nearly all patients in the sample over the relevant time period, in order to produce an accurate estimate of the numbers of patients served by each provider. Figure 6 shows counts of buprenorphine providers per month, according to those measures. The gap between the gold line (the number of prescribers serving patients with any insurance type) and the purple line (the number serving Medicaid patients) indicates that some buprenorphine prescribers are never matched with Medicaid patients (in our sample at least), although that does not necessarily reflect a deliberate policy to exclude such patients. On average, in the 2014-2015 period compared with the 2012-2013 period, the number of buprenorphine prescribers per month serving patients with any insurance type increased nearly 25 percent; the number per month prescribing to Medicaid patients increased by about 21 percent for the same period. Despite these gains, during the post-ACA period (January 2014 through December 2015) the net increase in prescriber stocks was less than the corresponding increase in the pre-ACA period (for either series), and the provider stocks actually edged lower in mid-2015. The second panel of Figure 6 shows that the average intensity of prescribing activity per Medicaid provider (in terms of number of patients per month) increased on average in the 20142015 period. Considering patients with any type of insurance, the average number of patients per provider increased about 30 percent in the 2014-2015 period compared with the pre-ACA period-from 12.5 patients to 16.3 patients per provider. Including only Medicaid patients, the average patient load increased 65 percent from the pre-ACA period to the post-ACA period, from 6.4 patients to 10.6 patients. The increase in Medicaid patients per provider was especially steep in the first few months of 2014. These patterns align with the fact that 2014 saw a large surge in the number of enrollees (recall Figure 1) and only a relatively modest increase in the number of buprenorphine providers.

The post-ACA increase in patient loads does not seem to have moved the average provider against their legal buprenorphine prescribing limits, which for most practitioners would have been 30 patients at any given time but could have been 100 patients for more experienced providers. Nonetheless, some providers might have approached or reached their effective capacity constraints, which, based on providers' resource limitations, may fall short of the legal limits. It is therefore possible that patients, including incumbents, faced longer wait times for appointments or found it harder to match initially with any provider starting in early 2014 . If so, 
however, these delays did not differ across county subdivisions according to the intensity of treatment in each place under the ACA.

\section{Discussion}

This study is the first we know of that describes the experience of incumbent Medicaid enrollees following implementation of the ACA in terms of their use of buprenorphine for OUD. Also, it is one of the first studies to describe changes in buprenorphine prescribing capacity under the ACA. It belongs to a small group of studies that consider post-ACA outcomes among incumbent Medicaid enrollees along any dimension of care. We find that the rate of buprenorphine receipt within a stable group of Medicaid incumbents generally increased from January 2012 to December 2015, although the pace of increase was slower in the post-ACA time period (January 2014 through December 2015) compared with the earlier period. If that aggregate pattern was in fact caused by the ACA, then any headwinds against buprenorphine utilization should arguably have been felt most acutely in the places in Rhode Island that experienced the greatest influxes of new enrollees under the ACA, all else being equal.

However, we find that Medicaid incumbents in county subdivisions (which correspond to cities or towns) that were subject to larger influxes of new (Medicaid or non-Medicaid) enrollees in 2014 and 2015 experienced roughly equivalent increases in their chances of receiving buprenorphine following implementation of the ACA. These results include controls for numerous other factors that predict buprenorphine receipt, such as county subdivision fixed effects, aggregate time effects, and individual health status indicators. In more robust models that (progressively) address the potential endogeneity of the actual enrollment increases, account for unobserved individual heterogeneity, and control for differing pre-ACA trends in buprenorphine use, again we find that the ACA implementation was effectively neutral for incumbent enrollees in terms of their chances of receiving buprenorphine. In sum, the regression results offer no support for claims that the ACA created problems for existing Medicaid enrollees in the states that expanded Medicaid, and so they would not help to make a case against expanding Medicaid in states that have not already done so.

To the extent that some models suggest that county subdivisions that were more intensively treated under the ACA (as predicted by their pre-ACA uninsurance rates) experienced larger gains in buprenorphine use following the ACA, the sensitivity analysis indicates that those 
results most likely reflect the fact that places with low uninsurance rates in the pre-ACA period (and that subsequently experienced smaller influxes of new non-Medicaid enrollees under the ACA) were simply experiencing slower growth in buprenorphine use both before and after the introduction of the ACA, for reasons not related to the ACA. For example, the slower growth in those places might have occurred because they had higher levels of buprenorphine use to begin with and therefore had less potential for growth in such use.

The potential benefits of health insurance expansions are thought to operate via a positive supply-side response (Finkelstein 2007; Meinhofer and Witman 2018). Regarding the supplyside response for buprenorphine prescribing in Rhode Island, the number of buprenorphine providers treating patients in our sample — both overall and the number treating Medicaid patients specifically_increased fairly steadily over the 2012-2014 period, but then it stabilized in 2015. The net increase in providers from the 2012-2013 period to the 2014-2105 period was modest in comparison with the overall surge in health insurance enrollees (of any insurance type) over the same time period. Accordingly, we observe that buprenorphine providers on average assumed higher patient loads starting in 2014, and in particular the number of Medicaid patients per provider increased markedly (not restricting to incumbent patients).

Whether these increased patient loads led to longer appointment wait times for incumbent patients can't be directly determined from our data. Nonetheless, our regression analysis suggests that any negative impact on incumbents (in terms of filling buprenorphine prescriptions) of an increase in patient loads per provider operated (if at all) only in the aggregate and does not align with our measures of treatment intensity under the ACA. The fact that providers took on more patients suggests that they had the capacity to do so. Indeed, even following the rise in patient loads after 2014, the average provider served at most 18 buprenorphine patients in a month, a number that falls well short of the typical legal patient limit of 30 per provider. On the other hand, providers may have been pushed closer to their effective capacity constraints - with potential negative fallout for patients. Those effective constraints are not observed, but they may fall short of the legal limits.

As noted above, a potential limitation of the approach concerns the geographic unit of analysis, which may be too small relative to the actual size of local markets for buprenorphine providers. A study of Massachusetts (Miller 2012) uses the county as the treatment unit, and this is a 
possible option for further research, but there are only five counties in Rhode Island. Another limitation is that we do not measure whether methadone use responded to Rhode Island's ACA implementation, nor how utilization of methadone and buprenorphine might have interacted with each other in the wake of the ACA. Although both medications have been found highly effective in treating OUD, research suggests that many patients have a preference for buprenorphine over methadone owing to its greater convenience and less stigmatized perception (Cioe et al. 2020), while methadone may be more effective for patients with very severe OUD. As the APCD data on methadone treatment have been found to be less reliable than the buprenorphine data, and the potential for expansion of MOUD has been focused on buprenorphine in recent years, the choice to focus on buprenorphine is well founded. Finally, as Rhode Island is a small state and because each state's implementation of the ACA and (where relevant) Medicaid expansion came with its own idiosyncrasies, the results may not be generalizable to the experience of Medicaid incumbents in other states.

\section{References}

American Psychiatric Association. 2013. Diagnostic and Statistical Manual of Mental Disorders (5th ed.). Washington, DC.

Antwi, Yaa Akosa, Asako S. Moriya, and Kosali I. Simon. 2015. “Access to Health Insurance and the Use of Inpatient Medical Care: Evidence from the Affordable Care Act Young Adult Mandate." Journal of Health Economics 39: 171-187. https://doi.org/10.1016/j.jhealeco.2014.11.007

Burke, Mary A. 2019. "Access to Medication-Assisted Treatment for Opioid Use Disorder: Is Rhode Island Different, and Why?" Federal Reserve Bank of Boston Current Policy Perspectives No. 19-2.

Burke, Mary A., and Riley Sullivan. 2020. "Medication-assisted Treatment for Opioid Use Disorder in Rhode Island: Who Gets Treatment, and Does Treatment Improve Health Outcomes?" Federal Reserve Bank of Boston. New England Public Policy Center Research Report 20-3.

Burke, Mary A., Riley Sullivan, Katherine Carman, J. Frank Wharam, Hefei Wen, and Hao Yu. 2021. "Who Gets Medication-assisted Treatment for Opioid Use Disorder, and Does It 
Reduce Overdose Risk? Evidence from the Rhode Island All-payer Claims Database." Research Department Working Papers No. 21-3. Boston, MA: Federal Reserve Bank of Boston.

Burns, Rachel M., Rosalie L. Pacula, Sebastian Bauhoff, Adam J. Gordon, Hollie Hendrikson, Douglas L. Leslie, and Bradley D. Stein. 2016. "Policies Related to Opioid Agonist Therapy for Opioid Use Disorders: The Evolution of State Policies from 2004 to 2013." Substance Abuse 37(1): 63-69. doi:10.1080/08897077.2015.1080208

Cioe, Katharine, Breanne E. Biondi, Rebecca Easly, Amanda Simard, Xiao Zheng, and Sandra A. Springer. 2020. “A Systematic Review of Patients' and Providers' Perspectives of Medications for Treatment of Opioid Use Disorder." Journal of Substance Abuse Treatment 119. https://doi.org/10.1016/j.jsat.2020.108146

Clemans-Cope, L., Epstein, M., and Kenney, G.M., 2017. "Rapid Growth in Medicaid Spending on Medications to Treat Opioid Use Disorder and Overdose.” Technical Report. Urban Institute.

Connery, Hilary Smith. 2015. "Medication-Assisted Treatment of Opioid Use Disorder: Review of Evidence and Future Directions." Harvard Review of Psychiatry 23(2): 63-75. Doi: 10.1097/HRP.0000000000000075

Courtemanche, Charles, James Marton, Benjamin Ukert, Aaron Yelowitz, and Daniela Zapata. 2017. "Early Impacts of the Affordable Care Act on Health Insurance Coverage in Medicaid Expansion and non-Expansion States. ”Journal of Policy Analysis and Management 36(1): 178-210.

Donohue, Julie, Peter Cunningham, Lauryn Walker, and Rachel Garfield. 2019. “Opioid Use Disorder among Medicaid Enrollees: Snapshot of the Epidemic and State Responses." Kaiser Family Foundation Issue Brief. November 15, 2019. https://www.kff.org/reportsection/opioid-use-disorder-among-medicaid-enrollees-snapshot-of-the-epidemic-and$\underline{\text { state-responses-issue-brief/ }}$ 
Finkelstein, Amy. 2007. "The Aggregate Effects of Health Insurance: Evidence from the Introduction of Medicare." The Quarterly Journal of Economics 122(1): 1-37. https://doi.org/10.1162/qjec.122.1.1.

Florence, Curtis, Feijun Luo, and Ketra Rice., 2021. "The Economic Burden of Opioid Use Disorder and Fatal Opioid Overdose in the United States, 2017." Drug and Alcohol Dependence 218: 108350. https://doi.org/10.1016/j.drugalcdep.2020.108350.

Gangopadhyaya, Anuj, and Emily M. Johnston. 2021. "Impacts of the ACA's Medicaid Expansion on Health Insurance Coverage and Health Care Access among Young Adults.” Washington, DC: Urban Institute.

Gibson, Amy, Louisa Degenhardt, Richard P. Mattick, Robert Ali, Jason White, and Susannah O’Brien. 2008. "Exposure to Opioid Maintenance Treatment Reduces Long-term Mortality." Addiction 103(3): 462-468. https://doi.org/10.1111/j.1360$\underline{0443.2007 .02090 . x}$

Green, Traci C., Jennifer Clarke, Lauren Brinkley-Rubinstein, Brandon D. L. Marshall, Nicole Alexander-Scott, Rebecca Boss, and Josiah D. Rich. 2018. "Postincarceration Fatal Overdoses after Implementing Medications for Addiction Treatment in a Statewide Correctional System.” JAMA Psychiatry 75(4): 405-407.

Doi: 10.1001/jamapsychiatry.2017.4614

Grooms, Jevay, and Alberto Ortega. 2019. "Examining Medicaid Expansion and the Treatment of Substance Use Disorders.” AEA Papers and Proceedings 109: 187-191. https://doi.org/10.1257/pandp.20191090

Holahan, John, Shanna Rifkin, Kevin Lucia, and Katie Keith. 2012., “ACA ImplementationMonitoring and Tracking: Rhode Island," Urban Institute. February 2012. https://www.urban.org/sites/default/files/publication/25091/412502-ACAImplementation-Monitoring-and-Tracking-Rhode-Island-Site-Visit-Report.PDF.

Kaestner, Robert, Bowen Garrett, Jiajia Chen, Anuj Gangopadhyaya, and Caitlyn Fleming. 2017. "Effects of ACA Medicaid Expansions on Health Insurance Coverage and Labor 
Supply." Journal of Policy Analysis and Management 36(3): 608-642.

https://doi.org/10.1002/pam.21993

Kaiser Family Foundation. 2020. "Opioid Overdose Death Rates and All Drug Overdose Death Rates per 100,000 Population (Age-Adjusted).” Kaiser Family Foundation. February 13, 2020.

Langabeer, James R., Angela L. Stotts, Arlene Cortez, Guillermo Tortolero, and Tiffany Champagne-Langabeer. 2020. "Geographic Proximity to Buprenorphine Treatment Providers in the US." Drug and Alcohol Dependence 213. https://doi.org/10.1016/j.drugalcdep.2020.108131

Larochelle, Marc R., Dana Bernson, Thomas Land, Thomas J. Stopka, Na Wang, Ziming Xuan, Sarah M. Bagley, Jane M. Liebschutz, and Alexander Y. Walley. 2018. "Medication for Opioid Use Disorder after Nonfatal Opioid Overdose and Association with Mortality." Annals of Internal Medicine 169(3): 137-145. Doi: 10.7326/M17-3107

Lipton, Brandy J., Sandra L. Decker, and Benjamin D. Sommers. 2017. "The Affordable Care Act Appears to Have Narrowed Racial and Ethnic Disparities in Insurance Coverage and Access to Care among Young Adults." Medical Care Research and Review 76(1): 32-55. https://doi.org/10.1177/1077558717706575

Maclean, Johanna Catherine, and Brendan Saloner. 2019. "The Effect of Public Insurance Expansions on Substance Use Disorder Treatment: Evidence from the Affordable Care Act." Journal of Policy Analysis and Management 38(2): 366-393. https://doi.org/10.1002/pam.22112

Mazurenko, Olena, Casey P. Bailo, Rajender Agarwal, Aaron E. Carroll, and Nir Menachemi. "The Effects of Medicaid Expansion Under The ACA: A Systematic Review." Health Affairs 37(6): 944-950. Doi: 10.1377/hlthaff.2017.1491

Meinhofer, Angélica, and Allison E. Witman, 2018. "The Role of Health Insurance on Treatment for Opioid Use Disorders: Evidence from the Affordable Care Act Medicaid Expansion." Journal of Health Economics 60: 177-197. https://doi.org/10.1016/j.jhealeco.2018.06.004 
Miller, Sarah. 2012. "The Effect of Insurance on Emergency Room Visits: An Analysis of the 2006 Massachusetts Health Reform.” Journal of Public Economics 96(11-12): 893-908. http://dx.doi.org/10.1016/j.jpubeco.2012.07.004

Miller, Sarah, and Laura R. Wherry. 2017. "Health and Access to Care during the First 2 Years of the ACA Medicaid Expansions." New England Journal of Medicine 376(10): 947-956.

Mohlman, Mary Kate, Beth Tanzman, Karl Finison, Melanie Pinette, and Craig Jones. 2016. "Impact of Medication-Assisted Treatment for Opioid Addiction on Medicaid Expenditures and Health Services Utilization Rates in Vermont." Journal of Substance Abuse Treatment 67: 9-14. http://dx.doi.org/10.1016/j.jsat.2016.05.002

Rhode Island Executive Office of Health and Human Services. 2012. Report to the Centers for Medicare and Medicaid Services. Quarterly Operation Report, Rhode Island Global Consumer Choice Compact, 1115 Waiver Demonstration.

Saloner, Brendan, and Shankar Karthikeyan. 2015. "Changes in Substance Abuse Treatment Use among Individuals with Opioid Use Disorders in the United States, 2004-2013." Journal of the American Medical Association 314(14): 1515-1517. Doi: 10.1001/jama.2015.10345

Schneider, Andy, Risa Elias, Rachel Garfield, David Rousseau, and Victoria Wachino. 2002. The Medicaid Resource Book. Washington, DC: Kaiser Commission on Medicaid and the Uninsured.

Schuckit, Marc A. 2016. "Treatment of Opioid-Use Disorders." New England Journal of Medicine 375: 357-368. Doi: 10.1056/NEJMra1604339

Simon, Kosali, Aparna Soni, and John Cawley. 2017. "The Impact of Health Insurance on Preventive Care and Health Behaviors: Evidence from the First Two Years of the ACA Medicaid Expansions." Journal of Policy Analysis and Management 36(2): 390417. https://doi.org/10.1002/pam.21972

Stein, Bradley D., Mark Sorbero, Andrew W. Dick, Rosalie Liccardo Pacula, Rachel M. Burns, and Adam J. Gordon. 2016. "Physician Capacity to Treat Opioid Use Disorder with 
Buprenorphine-Assisted Treatment." Journal of the American Medical Association 316(11): 1211-1212.

Volkow, Nora D., Thomas R. Frieden, Pamela S. Hyde, and Stephen S. Cha. 2014. "MedicationAssisted Therapies-Tackling the Opioid-Overdose Epidemic." New England Journal of Medicine 370(22): 2063-2066. Doi: 10.1056/NEJMp1402780

Wall, Ronald, Jurgem Rehm, Benedickt Fischer, Bruno Brands, Louis Gliksman, Jennifer Stewart, Wendy Medved, and Joan Blake. 2000. "Social Costs of Untreated Opioid Dependence." Journal of Urban Health: Bulletin of the New York Academy of Medicine. 77(4): 688-722. https://doi.org/10.1007/BF02344032

Wen, Hefei, Janet R. Cummings, Jason M. Hockenberry, Laura M. Gaydos, and Benjamin G. Druss. 2013. "State Parity Laws and Access to Treatment for Substance Use Disorder in the United States: Implications for Federal Parity Legislation." JAMA Psychiatry 70(12): $1355-1362$.

Wen, Hefei, Jason M. Hockenberry, Tyrone Borders, and Benjamin Druss. 2017. "Impact of Medicaid Expansion on Medicaid-covered Utilization of Buprenorphine for Opioid Use Disorder Treatment." Medical Care 55(4): 336-341.

Yarbrough, Courtney R., Amanda J. Abraham, and Grace Bagwell Adams. 2020. "Relationship of County Opioid Epidemic Severity to Changes in Access to Substance Use Disorder Treatment, 2009-2017.” Psychiatric Services 71: 12-20. https://doi.org/10.1176/appi.ps.201900150 
Figure 1: Unique Enrollees in the Medicaid Incumbent Analysis Sample and the Basic Sample

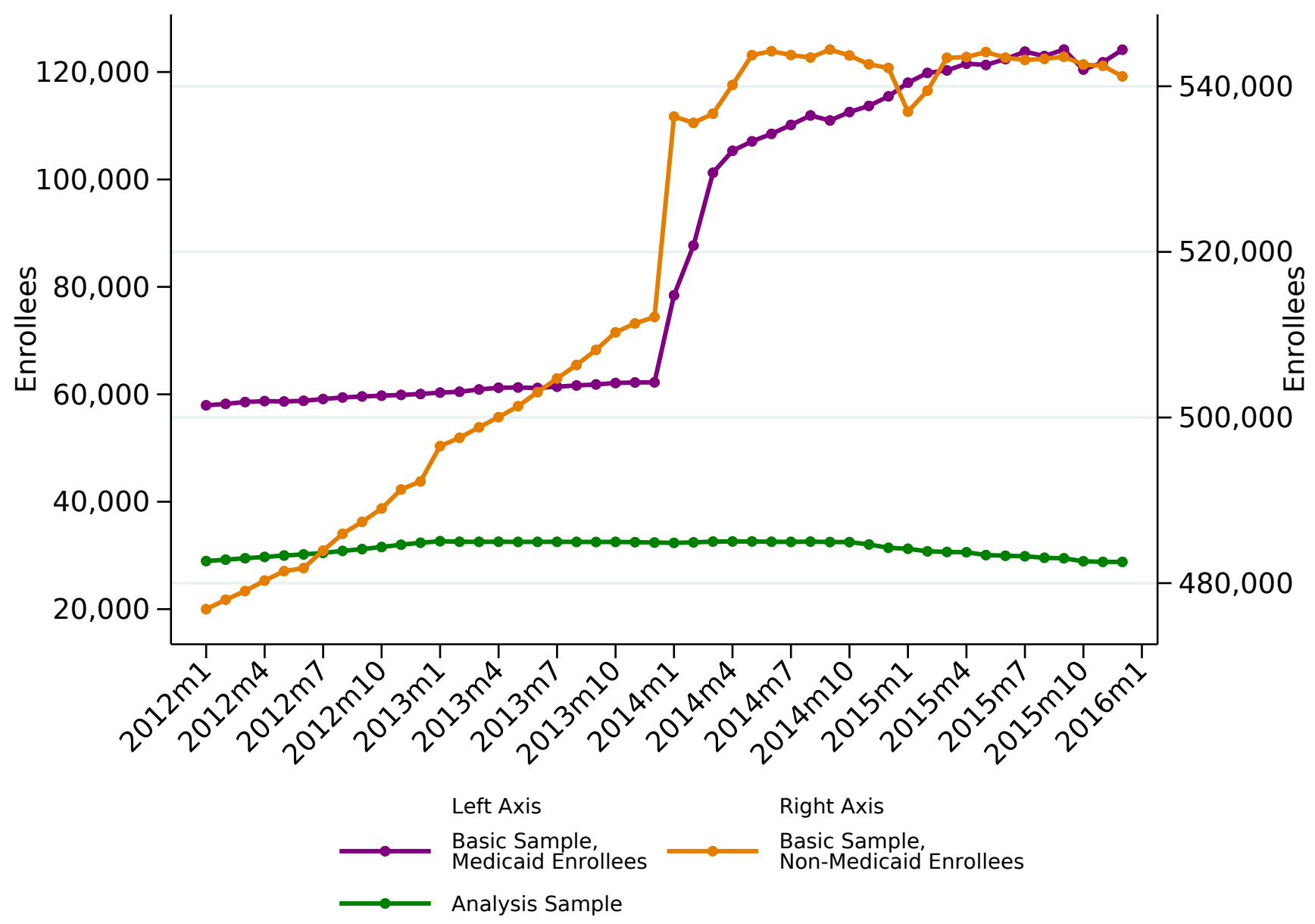

Source: Authors' calculations using data from HealthFacts RI.

Notes: The Medicaid Incumbent Analysis Sample consists of all Rhode Island residents aged 19 and older (as of January 2013) who were enrolled exclusively in Medicaid in all of their available observations dated from January 2012 through December 2014, and who were observed in at least 22 of the 24 months dated January 2013 through December 2014. For the selected set of individuals, all observations spanning January 2012 through December 2015 are used, provided the individual was enrolled in Medicaid in the given month. The Basic Sample requires that an enrollee have six consecutive observations (regardless of insurance type) in the APCD from April 2011 through May 2019, after imposing exclusions such as residing in Rhode Island and not exhibiting irregular age or gender changes. Medicaid enrollees do not include those dually eligible for Medicaid and Medicare. 
Table 1: Selected Sample Means for the Medicaid Incumbent Analysis Sample and the Basic Sample Medicaid Subset, 2012-2013

\begin{tabular}{r|cc}
\hline & Analysis Sample & Basic Sample Medicaid Subset \\
\hline Filled a Buprenorphine Prescription & 0.028 & 0.018 \\
& $(0.001)$ & $(0.000)$ \\
Opioid Use Disorder & 0.702 & 0.666 \\
& $(0.003)$ & $(0.002)$ \\
Alcohol Use Disorder & 0.072 & 0.045 \\
& $(0.001)$ & $(0.001)$ \\
Substance Use Disorder & 0.072 & 0.049 \\
& $(0.001)$ & $(0.001)$ \\
High-dose Opioid Prescription & 0.103 & 0.068 \\
& $(0.002)$ & $(0.001)$ \\
Opioid Overdose & 0.120 & 0.079 \\
& $(0.002)$ & $0.001)$ \\
Age as of Month Closest to January 2013 & $(0.000)$ & $(0.003$ \\
& 38 & 35 \\
Resides in Providence & $(0.069)$ & $(0.046)$ \\
& 0.322 & 0.308 \\
& $(0.003)$ & $(0.002)$ \\
\hline Unique Individuals & 32,654 & 79,175 \\
\hline
\end{tabular}

Source: Authors' calculations using data from HealthFacts RI and U.S. Census Bureau.

Notes: The Medicaid Incumbent Analysis Sample and the Basic Sample are defined in the Notes to Figure 1. The Medicaid subset of the basic sample consists of members of the basic sample who were enrolled in Medicaid in at least 3 months from January 2012 through December 2013. All values are sample means. Standard errors are in parentheses. Filled a Buprenorphine Prescription requires that an individual filled at least one buprenorphine prescription in 2012 or 2013. Opioid Use Disorder is defined as having had at least one diagnosis of either opioid abuse or opioid dependence (based on ICD-9 codes) in either 2012 or 2013. Similarly, for other conditions, at least one diagnosis in either 2012 or 2013 is required. Substance Use Disorder excludes opioids and alcohol. High-dose Opioid Prescription requires that an individual filled at least one high-dose opioid prescription (a dosage of more than 90 medical morphine equivalents per day) in 2012 or 2013, provided they filled at least two such prescriptions from 2011 through 2019. Resides in Providence requires having at least one observation in 2012 or 2013 in which the enrollee's county subdivision of residence is Providence. 
Figure 2: Buprenorphine Recipients per 100,000 Enrollees and Counterfactual Medicaid Incumbent Analysis Sample

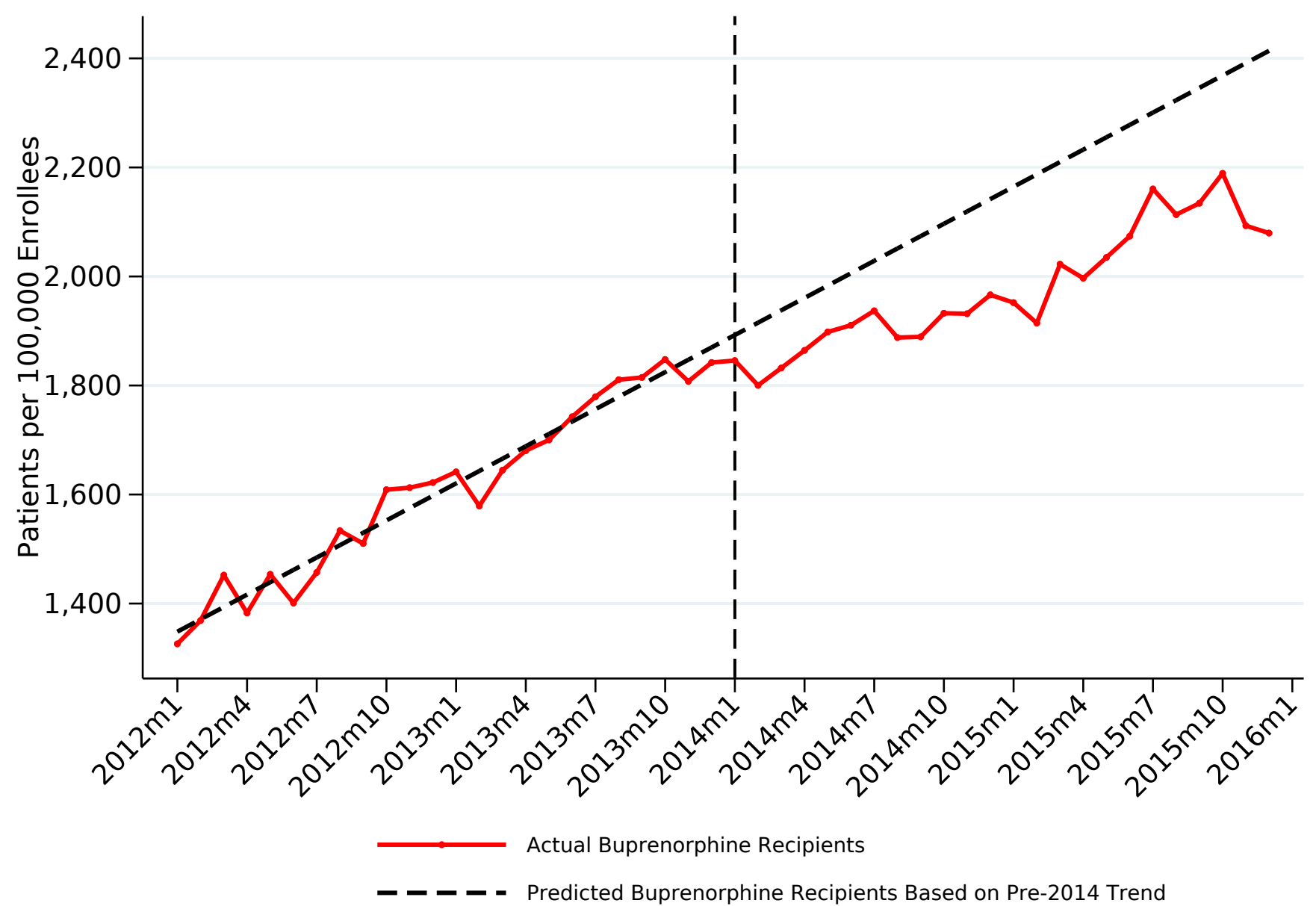

Source: Authors' calculations using data from HealthFacts RI.

Notes: The dashed diagonal line shows the predicted number of buprenorphine recipients based on the fitted linear trend in buprenorphine receipt for the 2012-2013 period. The dashed vertical line marks the month (January 2014) when the Affordable Care Act went into effect. 
Table 2: Mean Values of Regression Variables in the Medicaid Incumbent Analysis Sample

\begin{tabular}{|c|c|c|c|c|c|}
\hline & 2012 & 2013 & 2014 & 2015 & Total \\
\hline \multirow{4}{*}{ Gross Percentage Increase in Non-Medicaid Enrollment } & 0.0148 & 0.0174 & 0.0189 & 0.0206 & 0.0179 \\
\hline & $(0.000)$ & $(0.000)$ & $(0.000)$ & $(0.000)$ & $(0.000)$ \\
\hline & 6.591 & 6.230 & 10.735 & 5.015 & 7.193 \\
\hline & $(0.002)$ & $(0.003)$ & $(0.007)$ & $(0.003)$ & $(0.003)$ \\
\hline \multirow[t]{2}{*}{ Gross Percentage Increase in Medicaid Enrollment } & 12.66 & 9.59 & 42.07 & 7.597 & 18.26 \\
\hline & $(0.003)$ & $(0.002)$ & $(0.010)$ & $(0.002)$ & $(0.012)$ \\
\hline \multirow[t]{2}{*}{ Percentage Uninsured in County Subdivision (2009-2013) } & 18.63 & 18.65 & 18.62 & 18.574 & 18.62 \\
\hline & $(0.011)$ & $(0.011)$ & $(0.011)$ & $(0.011)$ & $(0.006)$ \\
\hline \multirow[t]{2}{*}{ Female } & 0.71 & 0.70 & 0.70 & 0.70 & 0.70 \\
\hline & $(0.001)$ & $(0.001)$ & $(0.001)$ & $(0.001)$ & $(0.000)$ \\
\hline \multirow[t]{2}{*}{ Alcohol Use Disorder (Ever) } & 0.17 & 0.17 & 0.17 & 0.18 & 0.17 \\
\hline & $(0.001)$ & $(0.001)$ & $(0.001)$ & $(0.001)$ & $(0.000)$ \\
\hline \multirow[t]{2}{*}{ Substance Use Disorder (Ever) } & 0.41 & 0.40 & 0.40 & 0.41 & 0.41 \\
\hline & $(0.001)$ & $(0.001)$ & $(0.001)$ & $(0.001)$ & $(0.000)$ \\
\hline \multirow[t]{2}{*}{ High-dose Opioid Prescription (Ever) } & 0.15 & 0.15 & 0.15 & 0.15 & 0.152 \\
\hline & $(0.001)$ & $(0.001)$ & $(0.001)$ & $(0.001)$ & $(0.000)$ \\
\hline \multirow[t]{2}{*}{ Opioid Overdose (Ever) } & 0.020 & 0.020 & 0.020 & 0.020 & 0.020 \\
\hline & $(0.000)$ & $(0.000)$ & $(0.000)$ & $(0.000)$ & $(0.000)$ \\
\hline \multirow[t]{2}{*}{ Aged $19-28$ as of 2013} & 0.25 & 0.26 & 0.26 & 0.26 & 0.26 \\
\hline & $(0.001)$ & $(0.001)$ & $(0.001)$ & $(0.001)$ & $(0.000)$ \\
\hline \multirow[t]{2}{*}{ Aged $29-36$ as of 2013} & 0.24 & 0.24 & 0.24 & 0.24 & 0.24 \\
\hline & $(0.001)$ & $(0.001)$ & $(0.001)$ & $(0.001)$ & $(0.000)$ \\
\hline \multirow[t]{2}{*}{ Aged $37-47$ as of 2013} & 0.26 & 0.26 & 0.26 & 0.26 & 0.26 \\
\hline & $(0.001)$ & $(0.001)$ & $(0.001)$ & $(0.001)$ & $(0.000)$ \\
\hline \multirow[t]{2}{*}{ Aged 48 or Older as of 2013} & 0.25 & 0.24 & 0.24 & 0.24 & 0.24 \\
\hline & $(0.001)$ & $(0.001)$ & $(0.001)$ & $(0.001)$ & $(0.000)$ \\
\hline Observations (Person-Months) & 366,001 & 390,336 & 388,713 & 358,710 & $1,503,760$ \\
\hline
\end{tabular}

Source: Authors' calculations using data from HealthFacts RI, U.S. Census Bureau, and American Community Survey.

Notes: Each value represents the sample mean of the given variable over all monthly observations in the given calendar year. Standard errors are in parentheses. Filled a Buprenorphine Prescription indicates that an individual filled at least one buprenorphine prescription in the month. Gross Percentage Increase in Medicaid/non-Medicaid Enrollment is defined as new enrollees of the given type (Medicaid or non-Medicaid) in the calendar year as a percentage of the previous year's enrollees of the same type. New enrollees must not have been observed in the APCD with any type of health insurance in any previous year. The Percentage Uninsured in County Subdivision (2009-2013) represents the average percentage (from 2009 through 2013) of adults aged 18 to 64 in the given county subdivision who lacked health insurance, calculated using five-year estimates from the 2013 American Community Survey (ACS). Alcohol Use Disorder (Ever) requires that an individual had at least one diagnosis of alcohol use disorder from 2011 through 2019. Substance Use Disorder (Ever) and Opioid Use Disorder (Ever) are defined similarly. Substance Use Disorder excludes opioids and alcohol. High-dose Opioid Prescription (Ever) requires that an individual filled at least two high-dose opioid prescriptions (a dosage of more than 90 medical morphine equivalents per day) from 2011 through 2019. 
Table 3: Baseline Logit Model of the Probability of Filling a Buprenorphine Prescription, Annual Enrollment Changes

Gross Percentage Increase in Non-Medicaid Enrollment by County Subdivision and Year

Gross Percentage Increase in Medicaid Enrollment by Country Subdivision and Year

(0.028)

$-0.011$

(0.014)

Year 2014 or $2015 \times$ Gross Percentage Increase in Non-Medicaid Enrollment by County Subdivision and Year

0.034

(0.022)

Year 2014 or $2015 \times$ Gross Percentage Increase in Medicaid Enrollment by Country Subdivision and Year

0.008

(0.015)

Female

$-0.048$

$(0.079)$

Alcohol Use Disorder (Ever)

$0.338^{* * *}$

Substance Use Disorder (Ever)

$(0.079)$

$2.700^{* * *}$

(0.143)

High-dose Opioid Prescription (Ever)

$-0.291^{* * *}$

(0.089)

Opioid Overdose (Ever)

$1.172^{* * *}$

(0.110)

$0.727^{* * *}$

Aged 29-36 as of 2013

(0.111)

Aged 37-47 as of 2013

$0.708^{* * *}$

(0.111)

Aged 48 or Older as of 2013

$0.351^{\text {*** }}$

(0.118)

Constant

$-7.687^{* * *}$

(0.204)

\begin{tabular}{lc}
\hline County Subdivision Effects & Yes \\
Month Effects & Yes \\
\hline Non-Medicaid Effect in Years 2014 and 2015 & 0.010 \\
& $(0.010)$ \\
Medicaid Effect in Years 2014 and 2015 & -0.003 \\
& $(0.003)$ \\
\hline Pseudo R-Squared & 0.173 \\
Observations & $1,503,760$ \\
\hline
\end{tabular}

Source: Authors' calculations using data from HealthFacts RI and U.S. Census Bureau.

Notes: Standard errors (in parentheses) are clustered by enrollee. See Notes to Table 2 for variable definitions. 
Figure 3: Fitted vs. Actual Probabilities of Receiving Buprenorphine by Selected County Subdivisions Predictions Based on Baseline Model

Providence-Example of "High Non-Medicaid Increase"
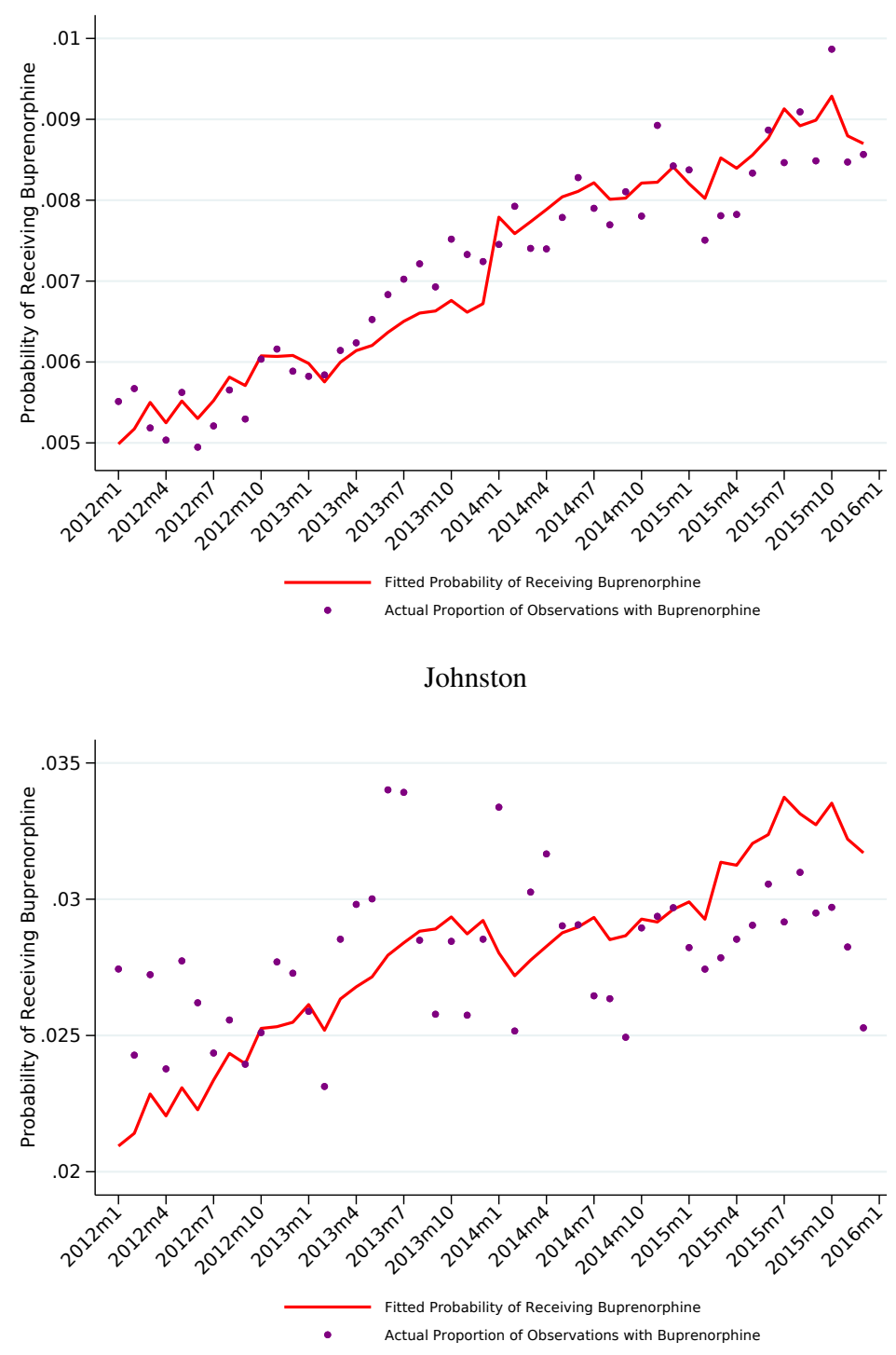

Warwick-Example of "Typical Medicaid/Non-Medicaid Increase"

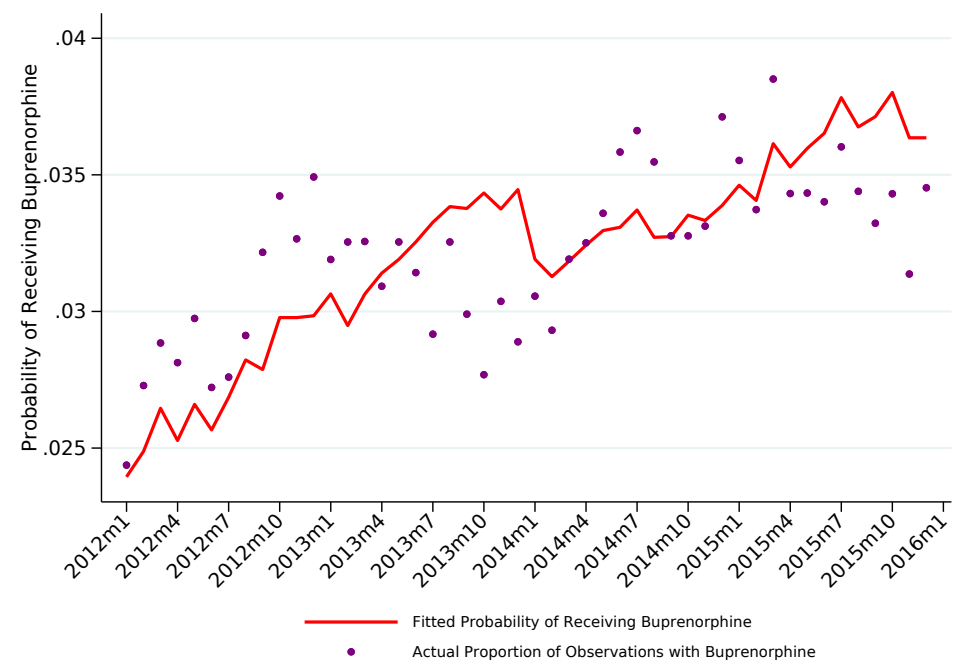

Cranston-Example of "High Medicaid Increase"

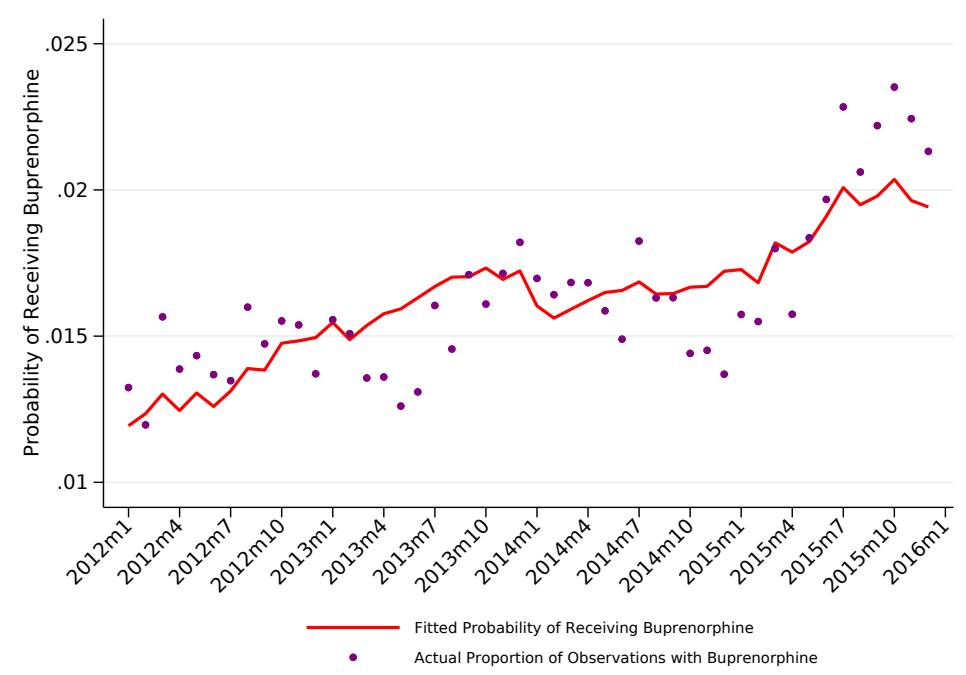


Figure 4: Percentage Change in Predicted Probability of Receiving Buprenorphine Pre- and Post-2014 Baseline Model

Ordered by 2014 Non-Medicaid Enrollment Increase

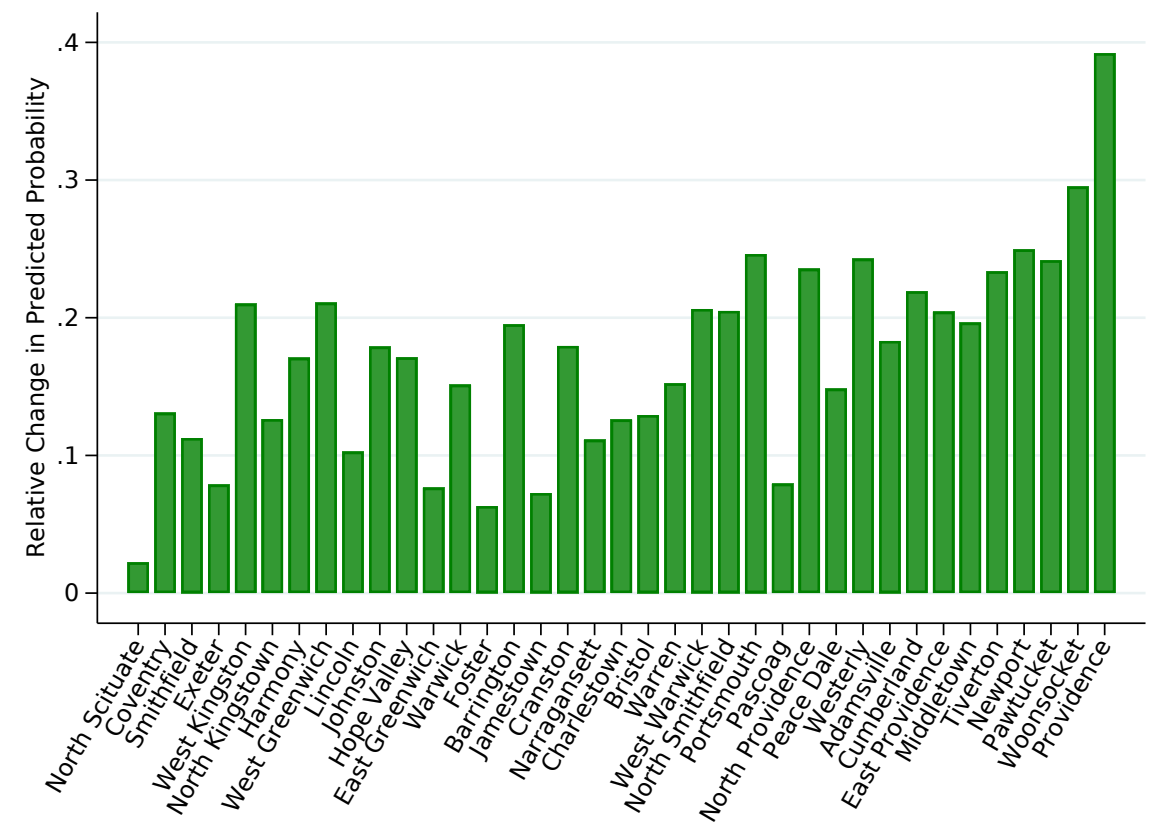

Ordered by 2014 Medicaid Enrollment Increase

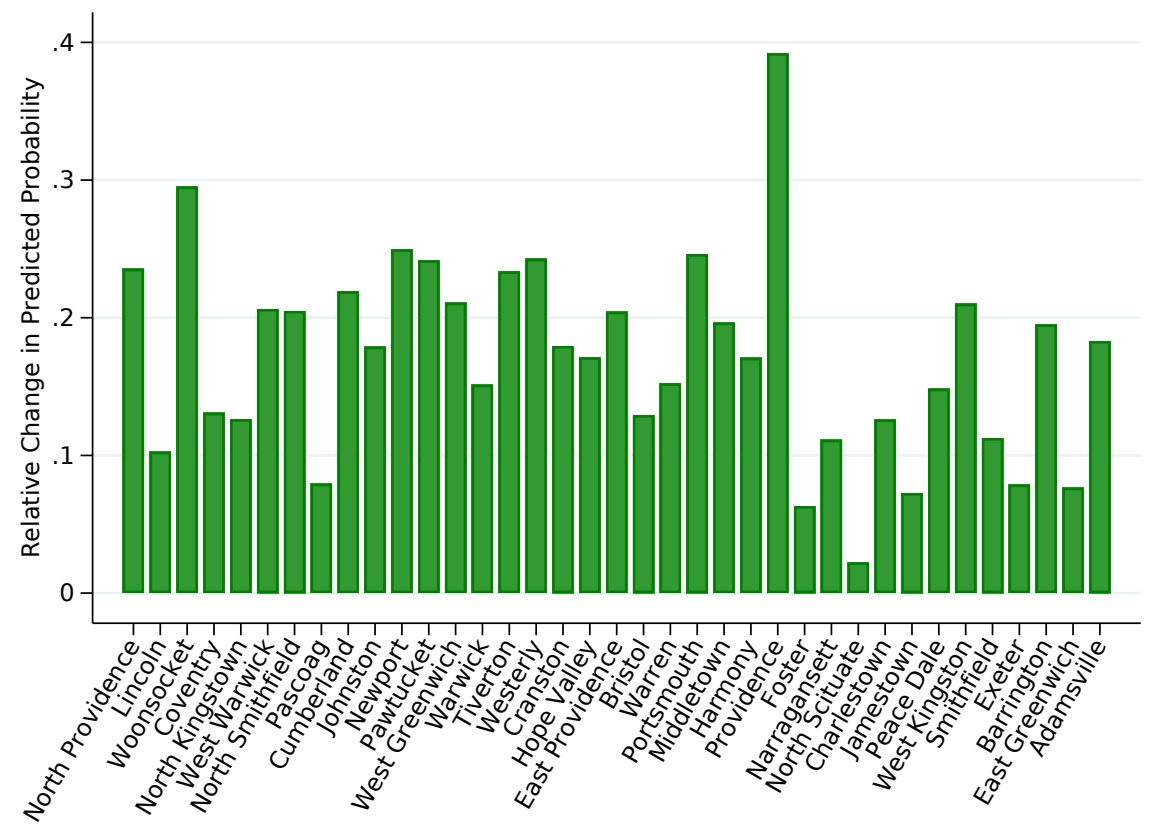

Source: Authors' calculations using data from HealthFacts RI and U.S. Census Bureau. 
Table 4: Alternative Logit Model of the Probability of Filling a Buprenorphine Prescription

\begin{tabular}{lc}
\hline & $(1)$ \\
\hline Year 2014 or $2015 \times$ Percentage Uninsured in County Subdivision $(2009-2013)$ & $0.011^{* * *}$ \\
Female & $(0.004)$ \\
& -0.048 \\
Alcohol Use Disorder (Ever) & $(0.079)$ \\
& $0.338^{* * *}$ \\
Substance Use Disorder (Ever) & $(0.079)$ \\
& $2.700^{* * *}$ \\
High-dose Opioid Prescription (Ever) & $(0.143)$ \\
Opioid Overdose (Ever) & $-0.291^{* * *}$ \\
Aged 29-36 as of 2013 & $(0.089)$ \\
Aged 37-47 as of 2013 & $1.172^{* * *}$ \\
& $(0.110)$ \\
Aged 48 or Older as of 2013 & $0.727^{* * *}$ \\
Constant & $(0.111)$ \\
& $0.708^{* * *}$ \\
County Subdivision Effects & $(0.111)$ \\
Month Effects & $0.351^{* * *}$ \\
\hline Pseudo R-Squared & $(0.118)$ \\
\hline Observations & $-7.678^{* * *}$ \\
\hline
\end{tabular}

Source: Authors' calculations using data from HealthFacts RI, U.S. Census Bureau, and American Community Survey.

Notes: Standard errors (in parentheses) are clustered by enrollee. See Notes to Table 2 for variable definitions. 
Figure 5: Fitted vs. Actual Probabilities of Receiving Buprenorphine by Selected County Subdivisions Predictions Based on Alternative Model

\section{Providence}

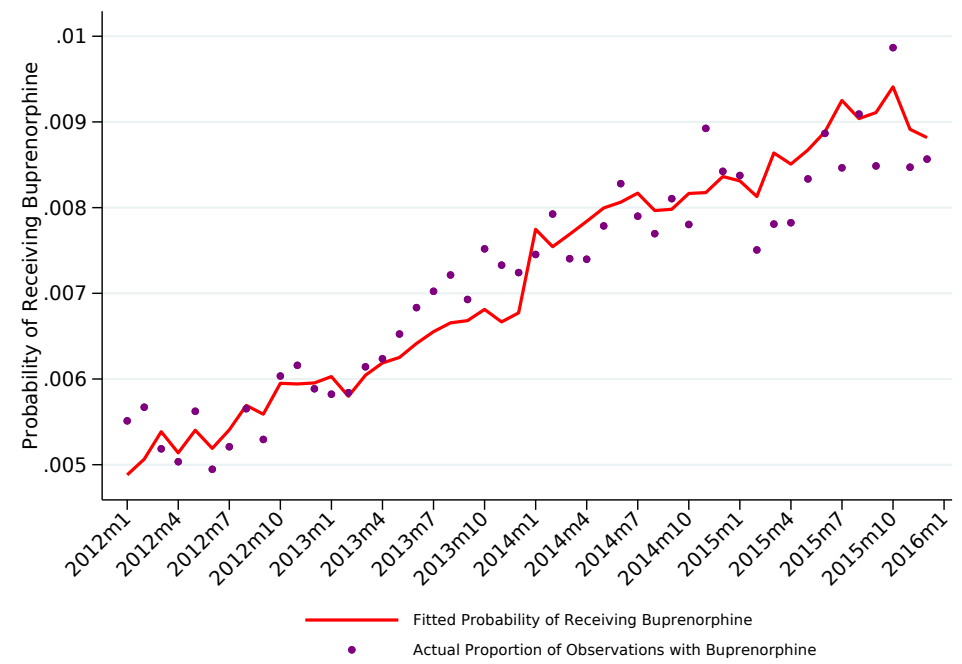

Johnston-Median of Sample Uninsured Share

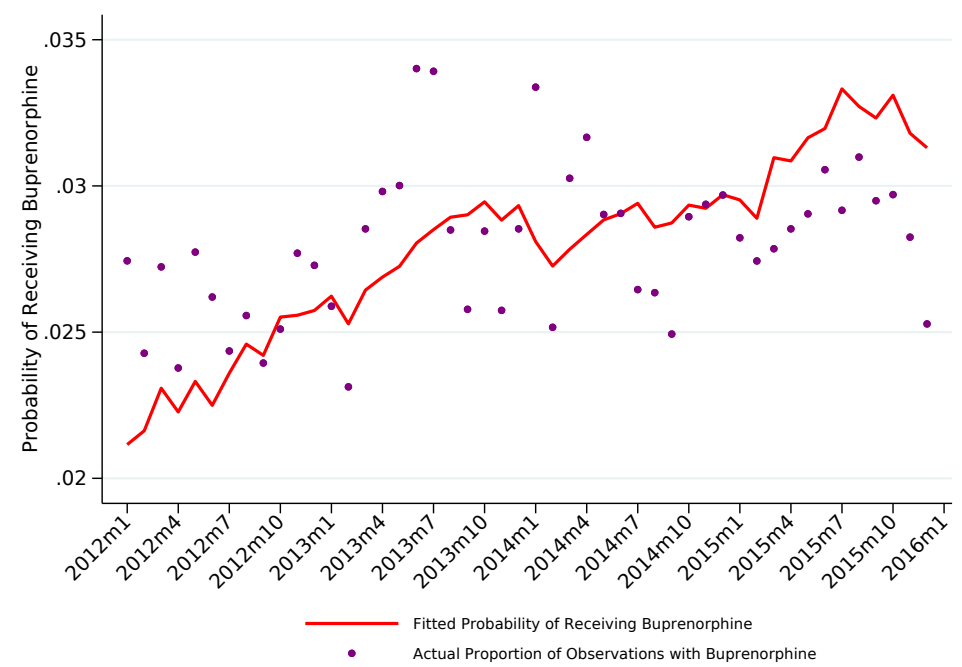

Warwick-25th Percentile of Sample Uninsured Share

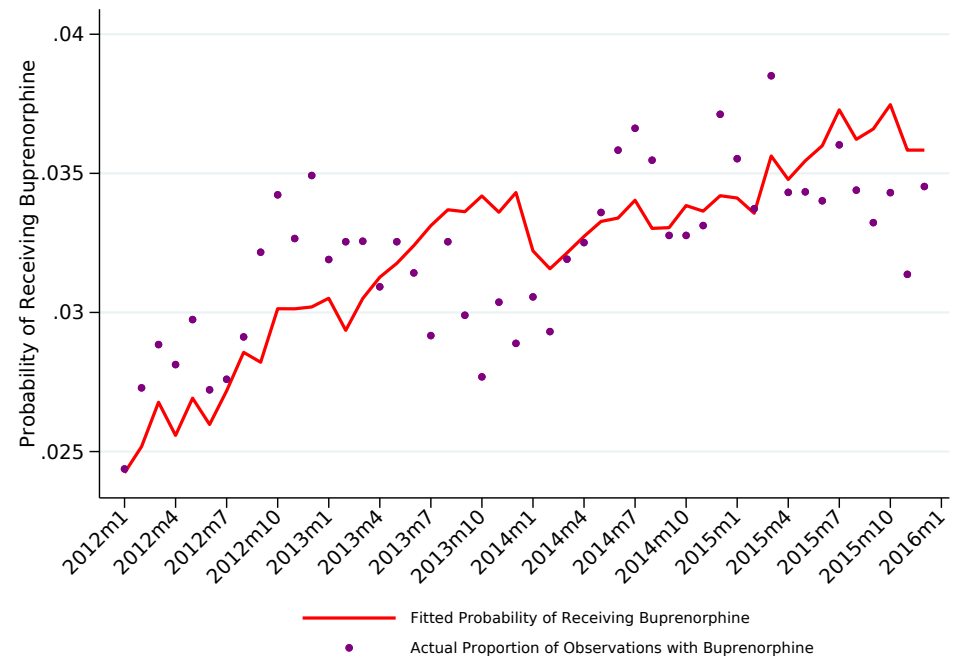

Cranston-75th Percentile of Sample Uninsured Share

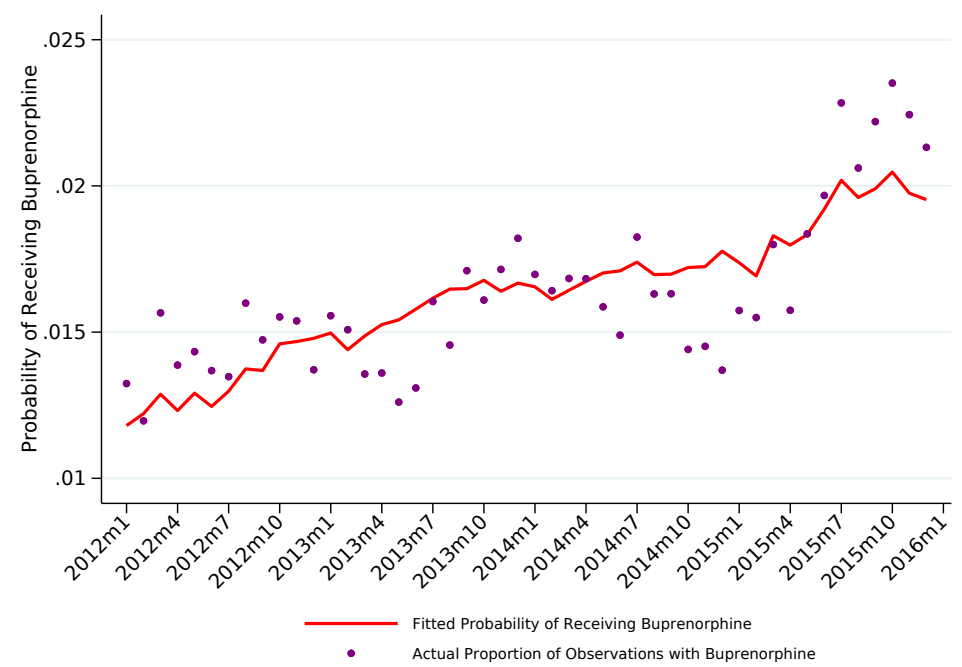

Source: Authors' calculations using data from HealthFacts RI, U.S. Census Bureau, and American Community Survey. 
Table 5: Conditional Logit (Fixed Effects) Model of the Probability of Filling a Buprenorphine Prescription

\begin{tabular}{lc}
\hline & $(1)$ \\
\hline Year 2014 or $2015 \times$ Percentage Uninsured in County Subdivision $(2009-2013)$ & $0.029^{* * *}$ \\
& $(0.004)$ \\
High-dose Opioid Prescription in Current Month & $-2.077^{* * *}$ \\
& $(0.095)$ \\
Opioid Overdose in Current Month & $-0.461^{* *}$ \\
& $(0.217)$ \\
\hline County Subdivision Effects & Yes \\
Month Effects & Yes \\
Individual Effects & Yes \\
\hline Observations & 54,597
\end{tabular}

Source: Authors' calculations using data from HealthFacts RI, U.S. Census Bureau, and American Community Survey.

Notes: Standard errors are in parentheses. See Notes to Table 2 for the definition of Percentage Uninsured in County Subdivision (2009-2013). High-dose Opioid Prescription in Current Month requires that an individual filled at least one high-dose opioid prescription (a dosage of more than 90 medical morphine equivalents per day) in the given month, provided they filled at least two such prescriptions from 2011 through 2019. Opioid Overdose in Current Month requires that an individual had at least one diagnosis of opioid overdose in the given month. 
Figure 6: Buprenorphine Prescriber Stocks and Average Buprenorphine Patient Loads

Stock of Prescribers

by Prescriber Type

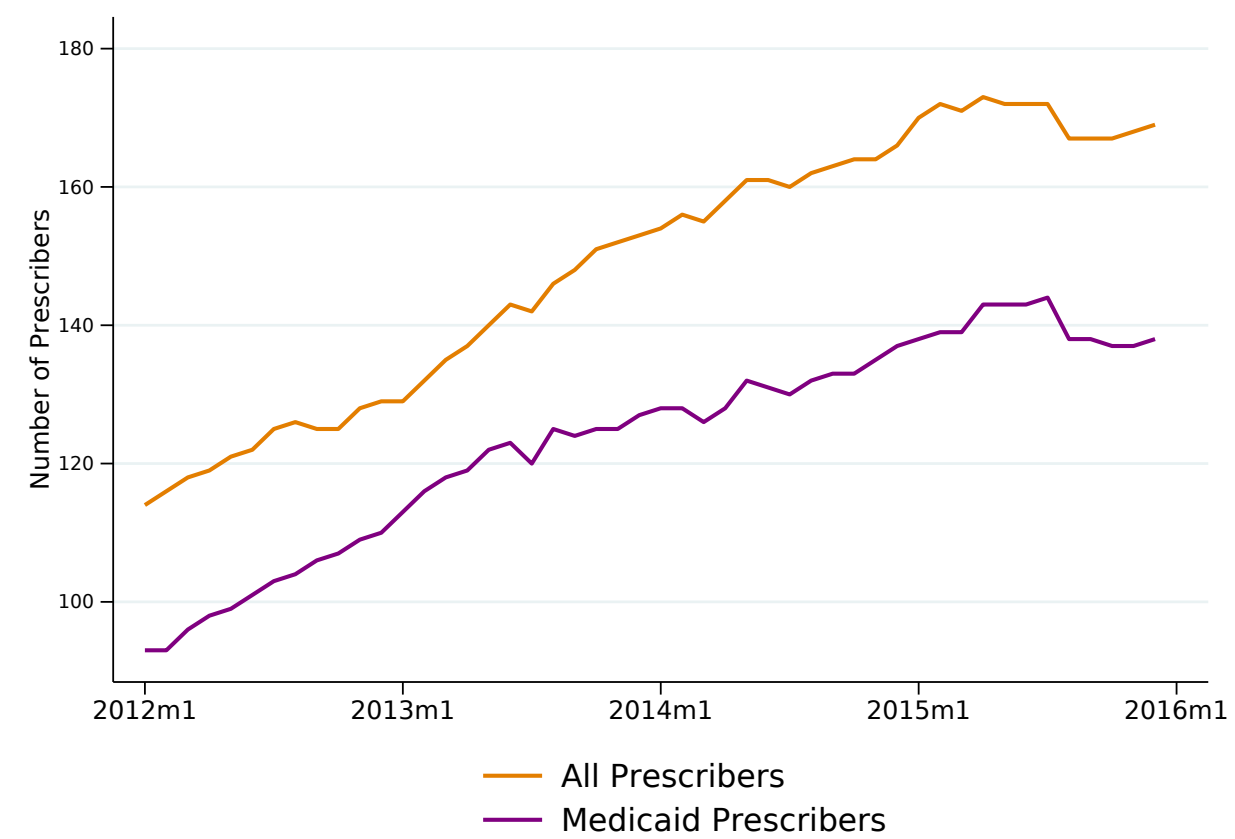

Average Number of Buprenorphine Patients per Prescriber by Patient and Prescriber Type

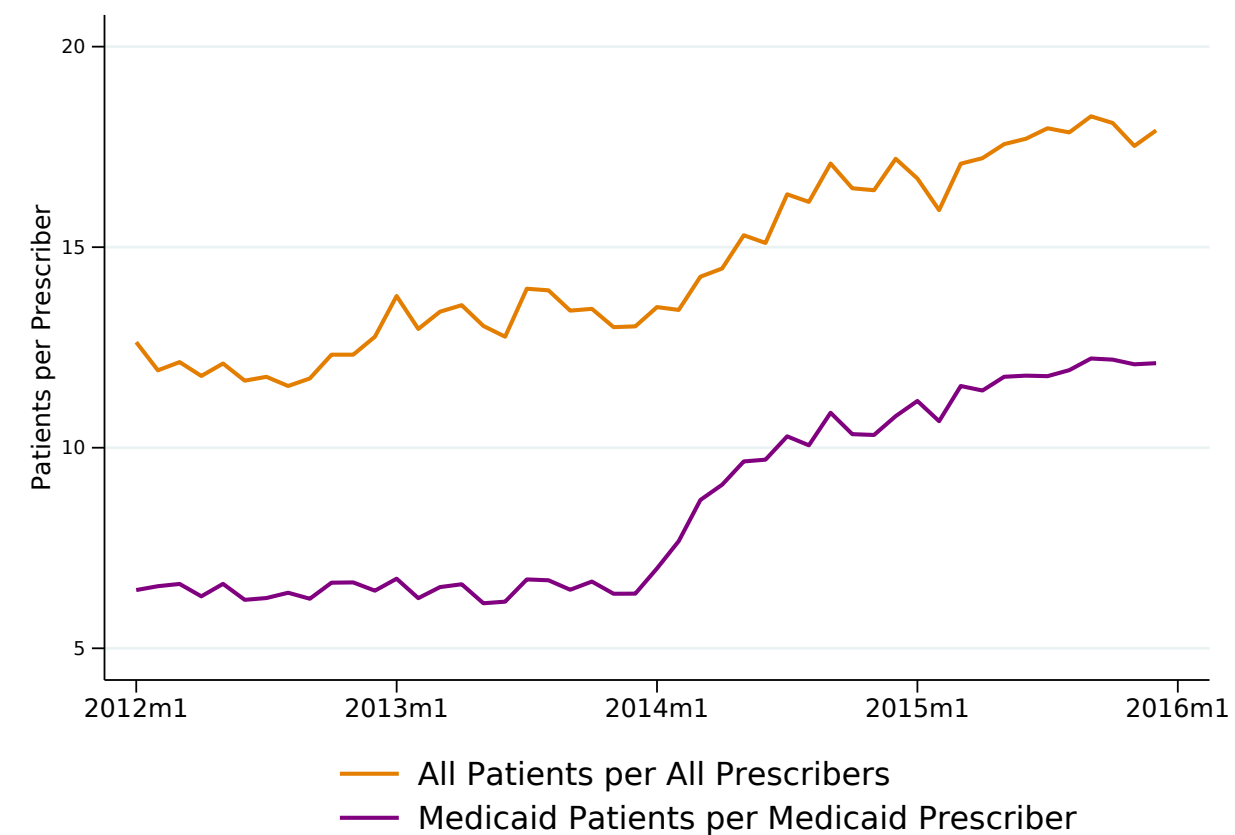

Source: Authors' calculations using data from HealthFacts RI.

Notes: All Prescribers refers to practitioners who ever prescribed buprenorphine to anyone, regardless of their health insurance type. Medicaid Prescribers refers to practitioners who ever prescribed to at least one Medicaid patient. All Patients refers to buprenorphine recipients with any pharmacy insurance type, and Medicaid Patients refers to buprenorphine recipients with Medicaid as their pharmacy insurance. The monthly stock of prescribers of a given type includes only those who have not exited from buprenorphine prescribing (to patients of the given type) as of the given month. Such exit refers to having a spell of at least six months with no buprenorphine prescriptions and writing no subsequent prescriptions after that spell. Moreover, all prescribers must have a credential code and be associated with a physical Rhode Island address. One-time prescribers are excluded. Patients with prescriptions from more than one provider are counted toward each prescriber's patient load. 
Figure A1: Gross Percentage Increase in Medicaid/Non-Medicaid Enrollment (APCD 2014) against Percentage Uninsured (ACS 2009-2013) by County Subdivision

Non-Medicaid Enrollment Increase

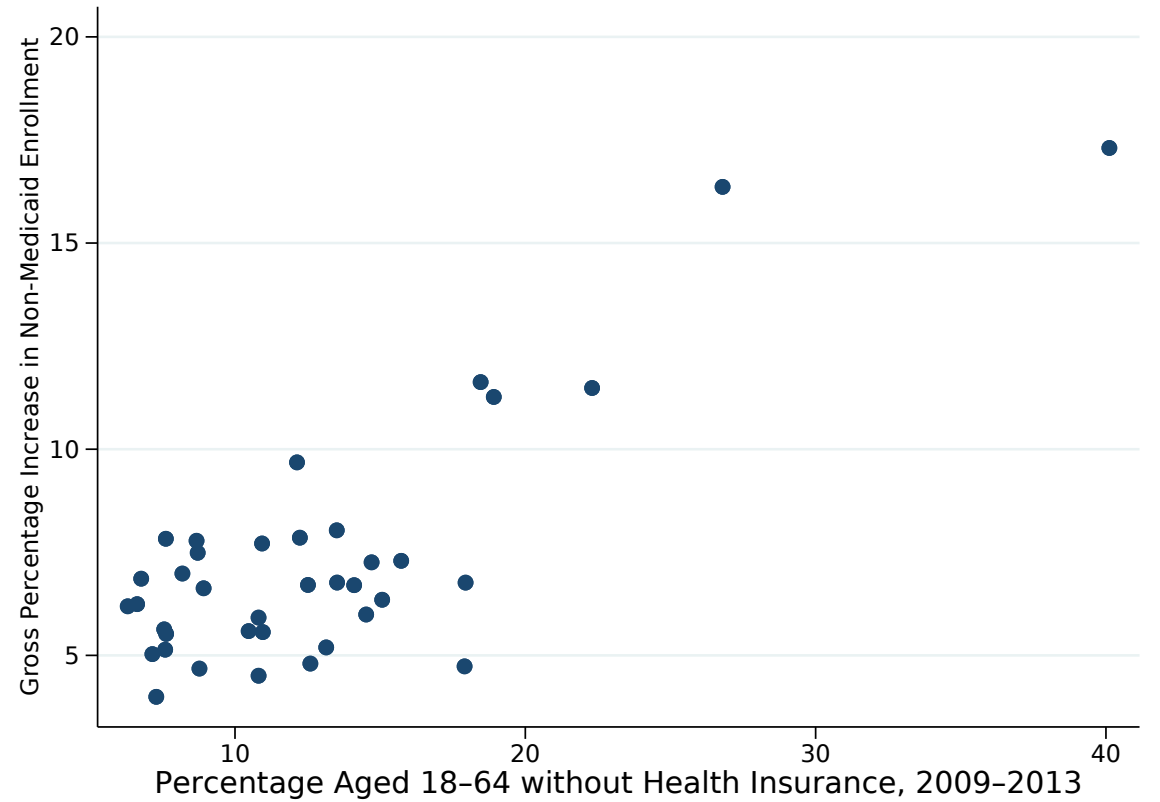

Medicaid Enrollment Increase

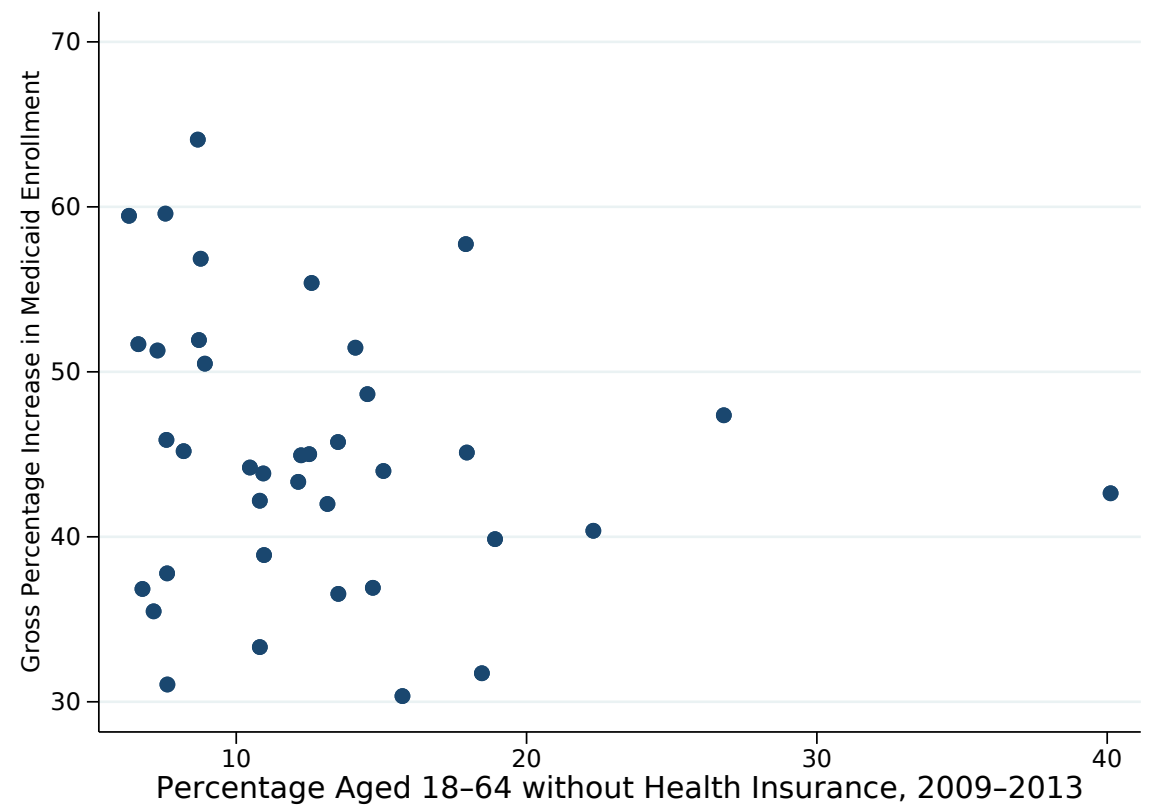

Source: Authors' calculations using data from HealthFacts RI, U.S. Census Bureau, and American Community Survey. Notes: See Notes to Table 2 for variable definitions. Block Island is excluded. 


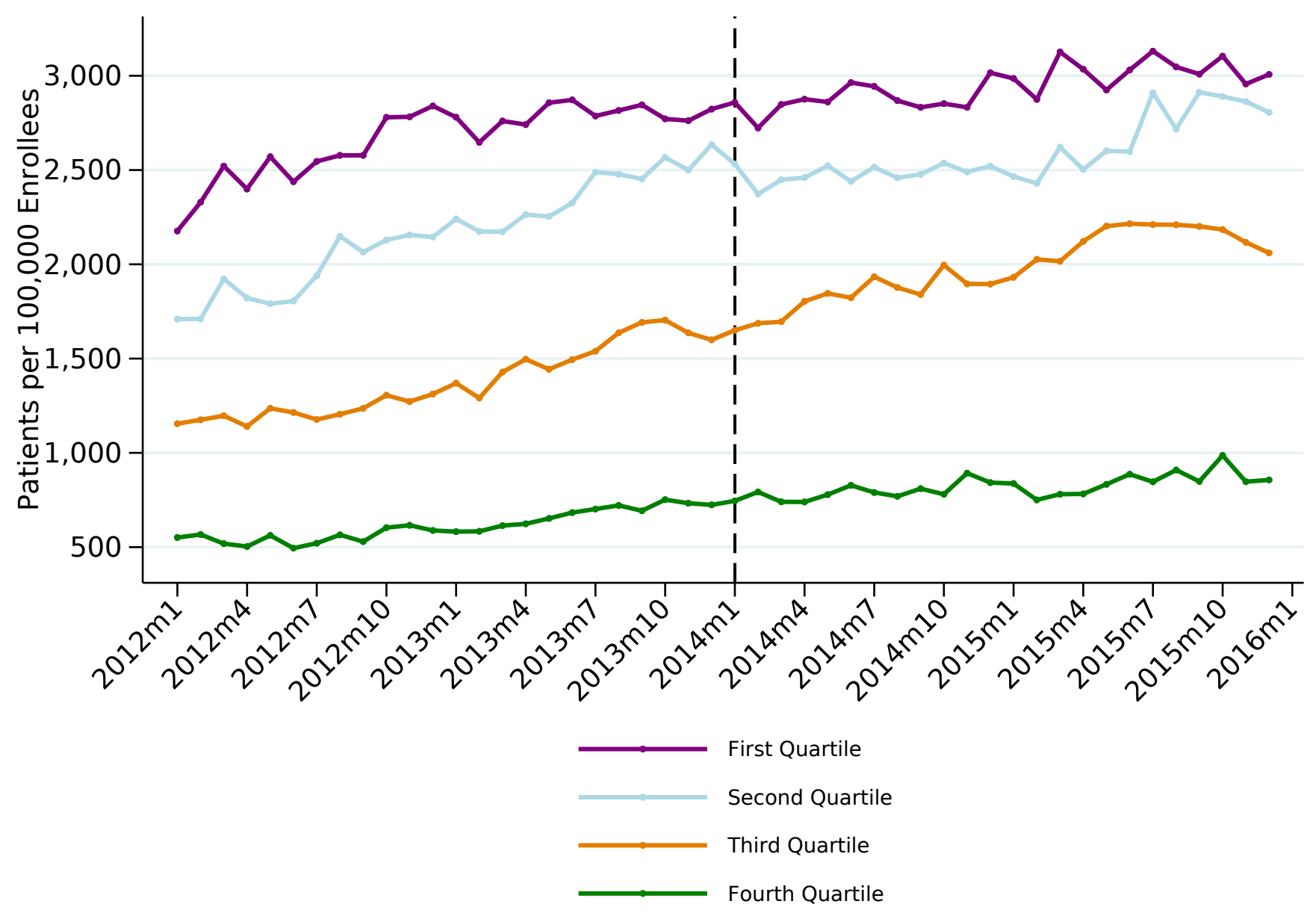

Source: Authors' calculations using data from HealthFacts RI, U.S. Census Bureau, and American Community Survey. Notes: Percentage Uninsured Quartiles refer to the quartiles of the Percentage Uninsured in County Subdivision (2009-2013). These weighted quartiles are identified using the sample observations from 2014 only, and then the appropriate quartile indicator is assigned to each observation. These indicators are fixed within a county subdivision across all time periods. Buprenorphine Recipients per 100,000 Enrollees is then calculated separately by month and quartile. 
Figure A3: Buprenorphine Recipients per 100,000 Enrollees within Gross Non-Medicaid Percentage Increase Quartiles

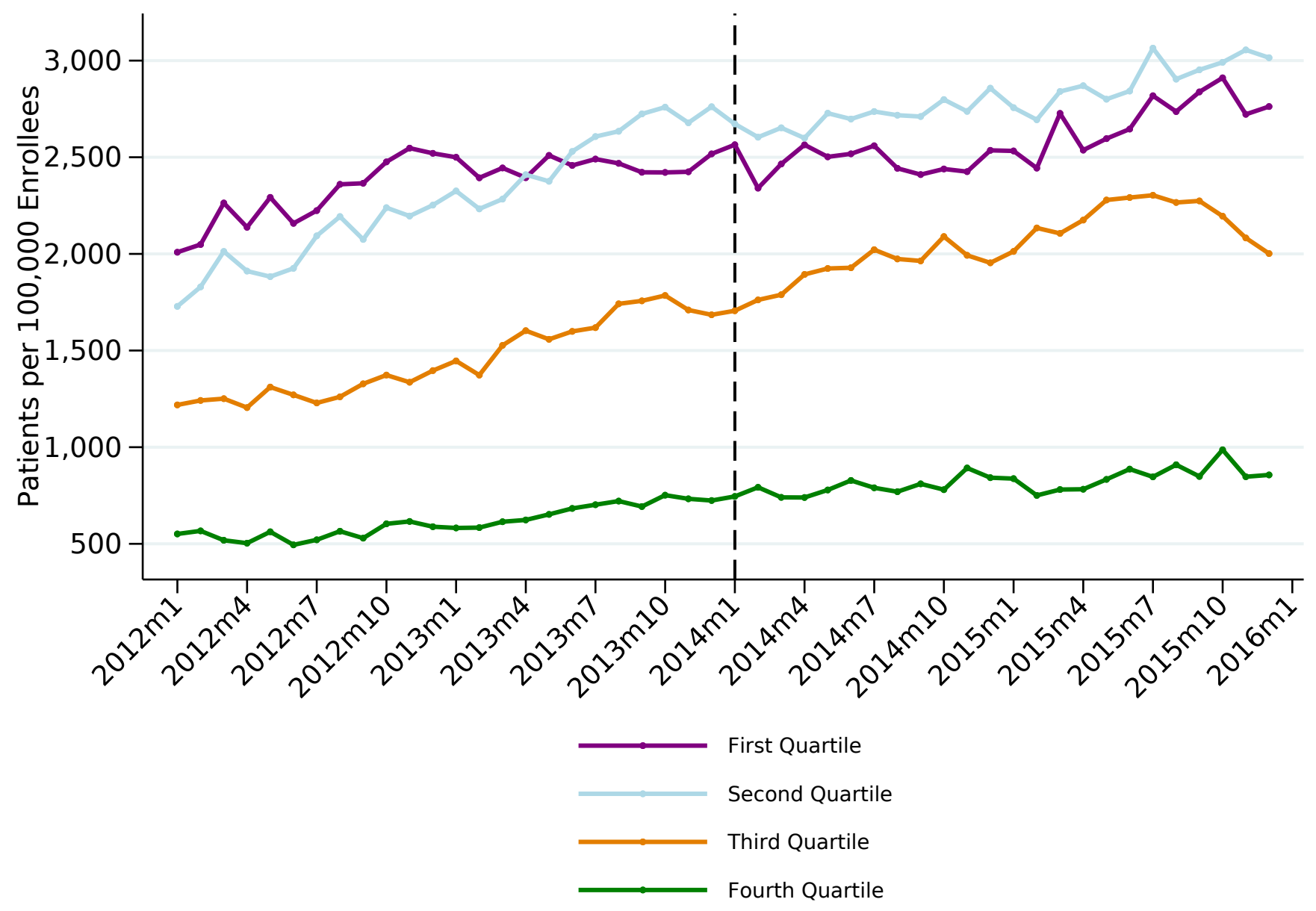

Source: Authors' calculations using data from HealthFacts RI and U.S. Census Bureau.

Notes: Gross Non-Medicaid Percentage Increase Quartiles refer to the quartiles of the Gross Non-Medicaid Percentage Increase by County Subdivision in 2014. These weighted quartiles are identified using the sample observations from 2014 only, and then the appropriate quartile indicator is assigned to each observation. These indicators are fixed within a county subdivision across all time periods. Buprenorphine Recipients per 100,000 Enrollees is then calculated separately by month and quartile. 
Figure A4: Buprenorphine Recipients per 100,000 Enrollees within Gross Medicaid Percentage Increase Quartiles

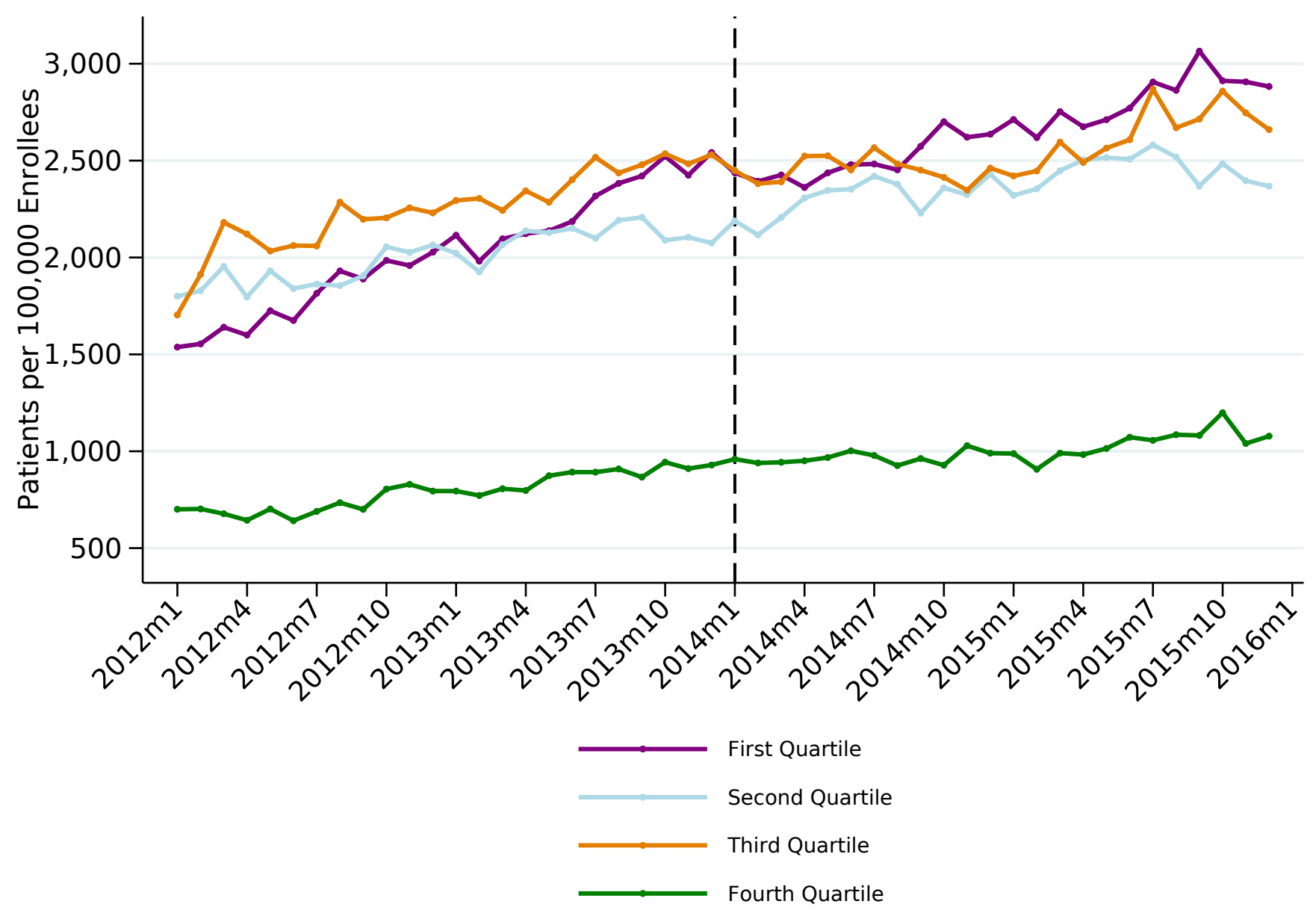

Source: Authors' calculations using data from HealthFacts RI and U.S. Census Bureau.

Notes: Gross Medicaid Percentage Increase Quartiles refer to the quartiles of the Gross Medicaid Percentage Increase by County Subdivision in 2014. These weighted quartiles are identified using the sample observations from 2014 only, and then the appropriate quartile indicator is assigned to each observation. These indicators are fixed within a county subdivision across all time periods. Buprenorphine Recipients per 100,000 Enrollees is then calculated separately by month and quartile. 
Table A1: Logit Tests for Pre-2014 Trends in Probability of Receiving Buprenorphine

\begin{tabular}{|c|c|c|c|}
\hline & $\begin{array}{c}\text { (1) } \\
\text { Prob. Bup. }\end{array}$ & $\begin{array}{c}\text { (2) } \\
\text { Prob. Bup. }\end{array}$ & $\begin{array}{c}\text { (3) } \\
\text { Prob. Bup. }\end{array}$ \\
\hline Monthly Trend & $\begin{array}{c}0.019^{* * *} \\
(0.004)\end{array}$ & $\begin{array}{c}0.018^{* * *} \\
(0.003)\end{array}$ & $\begin{array}{c}0.019^{* * *} \\
(0.004)\end{array}$ \\
\hline Monthly Trend $\times$ First Quartile of Percentage Uninsured & $\begin{array}{c}-0.010^{* *} \\
(0.004)\end{array}$ & & \\
\hline Monthly Trend $\times$ Second Quartile of Percentage Uninsured & $\begin{array}{c}0.001 \\
(0.005)\end{array}$ & & \\
\hline Monthly Trend $\times$ Third Quartile of Percentage Uninsured & $\begin{array}{c}0.001 \\
(0.005)\end{array}$ & & \\
\hline Monthly Trend $\times$ First Quartile of Percentage Medicaid Increase & & $\begin{array}{c}0.005 \\
(0.004)\end{array}$ & \\
\hline Monthly Trend $\times$ Second Quartile of Percentage Medicaid Increase & & $\begin{array}{c}-0.009^{* *} \\
(0.004)\end{array}$ & \\
\hline Monthly Trend $\times$ Third Quartile of Percentage Medicaid Increase & & $\begin{array}{l}-0.005 \\
(0.004)\end{array}$ & \\
\hline Monthly Trend $\times$ First Quartile of Percentage Non-Medicaid Increase & & & $\begin{array}{c}-0.011^{* *} \\
(0.004)\end{array}$ \\
\hline Monthly Trend $\times$ Second Quartile of Percentage Non-Medicaid Increase & & & $\begin{array}{c}0.002 \\
(0.005)\end{array}$ \\
\hline Monthly Trend $\times$ Third Quartile of Percentage Non-Medicaid Increase & & & $\begin{array}{c}0.001 \\
(0.005)\end{array}$ \\
\hline Female & $\begin{array}{c}-0.086^{* * *} \\
(0.020)\end{array}$ & $\begin{array}{c}-0.086^{* * *} \\
(0.020)\end{array}$ & $\begin{array}{c}-0.086^{* * *} \\
(0.020)\end{array}$ \\
\hline Alcohol Use Disorder (Ever) & $\begin{array}{c}0.314^{* * *} \\
(0.020)\end{array}$ & $\begin{array}{c}0.314^{* * *} \\
(0.020)\end{array}$ & $\begin{array}{c}0.314^{* * *} \\
(0.020)\end{array}$ \\
\hline Substance Use Disorder (Ever) & $\begin{array}{c}2.812^{* * *} \\
(0.037)\end{array}$ & $\begin{array}{c}2.812^{* * *} \\
(0.037)\end{array}$ & $\begin{array}{c}2.812^{* * *} \\
(0.037)\end{array}$ \\
\hline High-dose Opioid Prescription (Ever) & $\begin{array}{c}-0.440^{* * *} \\
(0.025)\end{array}$ & $\begin{array}{c}-0.440^{* * *} \\
(0.025)\end{array}$ & $\begin{array}{c}-0.440^{* * *} \\
(0.025)\end{array}$ \\
\hline Opioid Overdose (Ever) & $\begin{array}{c}1.223^{* * *} \\
(0.031)\end{array}$ & $\begin{array}{c}1.223^{* * *} \\
(0.031)\end{array}$ & $\begin{array}{c}1.223^{* * *} \\
(0.031)\end{array}$ \\
\hline Aged 28 or Younger as of 2013 & $\begin{array}{c}-0.504^{* * *} \\
(0.032)\end{array}$ & $\begin{array}{c}-0.504^{* * *} \\
(0.032)\end{array}$ & $\begin{array}{c}-0.504^{* * *} \\
(0.032)\end{array}$ \\
\hline Aged $29-36$ as of 2013 & $\begin{array}{c}0.346^{* * *} \\
(0.026)\end{array}$ & $\begin{array}{c}0.346^{* * *} \\
(0.026)\end{array}$ & $\begin{array}{c}0.346^{* * *} \\
(0.026)\end{array}$ \\
\hline Aged $37-47$ as of 2013 & $\begin{array}{c}0.326^{* * *} \\
(0.025)\end{array}$ & $\begin{array}{c}0.326^{* * *} \\
(0.025)\end{array}$ & $\begin{array}{c}0.326^{* * *} \\
(0.025)\end{array}$ \\
\hline Constant & $\begin{array}{c}-7.308^{* * *} \\
(0.067)\end{array}$ & $\begin{array}{c}-7.298^{* * *} \\
(0.061)\end{array}$ & $\begin{array}{c}-7.308^{* * *} \\
(0.067)\end{array}$ \\
\hline County Subdivision Effects & Yes & Yes & Yes \\
\hline Observations & 755,478 & 755,478 & 755,478 \\
\hline
\end{tabular}

Source: Authors' calculations using data from HealthFacts RI, U.S. Census Bureau, and American Community Survey.

Notes: Robust standard errors are in parentheses. Percentage Uninsured Quartiles refer to the quartiles of the Percentage Uninsured in County Subdivision (2009-2013). These weighted quartiles are identified using the sample observations from 2014 only, and then the appropriate quartile indicator is assigned to each observation. These indicators are fixed within a county subdivision across all time periods. Percentage Medicaid/Non-Medicaid Increase Quartiles are defined similarly. In all models, the fourth quartile of the relevant variable is omitted. See Notes to Table 2 for other variable definitions. 
Table A2: Sensitivity Analyses for Models of the Probability of Filling a Buprenorphine Prescription, Omitting Non-conforming Quartiles

\begin{tabular}{|c|c|c|c|c|}
\hline & (1) & (2) & (3) & (4) \\
\hline Year 2014 or $2015 \times$ Gross Percentage Increase in Non-Medicaid Enrollment by County Subdivision and Year & $\begin{array}{c}0.027 \\
(0.026)\end{array}$ & $\begin{array}{c}0.026 \\
(0.024)\end{array}$ & & \\
\hline Year 2014 or $2015 \times$ Gross Percentage Increase in Medicaid Enrollment by Country Subdivision and Year & $\begin{array}{l}-0.012 \\
(0.018)\end{array}$ & $\begin{array}{c}0.013 \\
(0.017)\end{array}$ & & \\
\hline Year 2014 or $2015 \times$ Percentage Uninsured in County Subdivision (2009-2013) & & & $\begin{array}{c}0.005 \\
(0.006)\end{array}$ & $\begin{array}{c}0.009 \\
(0.006)\end{array}$ \\
\hline County Subdivision Effects & Yes & Yes & Yes & Yes \\
\hline Month Effects & Yes & Yes & Yes & Yes \\
\hline Individual Effects & No & No & No & Yes \\
\hline Observations & $1,132,551$ & $1,140,412$ & $1,172,695$ & 37,098 \\
\hline
\end{tabular}

Source: Authors' calculations using data from HealthFacts RI, U.S. Census Bureau, and American Community Survey.

Notes: Column 1 shows results for a version of the baseline model that drops observations associated with the first quartile of the Gross Percentage Increase in Non-Medicaid Enrollment in 2014. Column 2 shows results for a version of the baseline model that drops observations associated with the second quartile of the Gross Percentage Increase in Medicaid Enrollment in 2014. Columns 3 and 4 show results for versions of the alternative and conditional logit models, respectively, that drop observations associated with the first quartile of Percentage Uninsured in County Subdivision (2009-2013). The table shows only the coefficients on the interaction terms of interest. See Table 3 for the list of other regressors included in the models in columns 1 and 2. See Tables 4 and 5, respectively, for the lists of other regressors included in columns 3 and 4 . See Notes to Table A1 for a description of how the quartiles of the relevant variables are calculated, and Notes to Table 2 for the definitions of the Gross Percentage Increase in Non-Medicaid/Medicaid Enrollment and the Percentage Uninsured. 